\title{
Computational Approaches and Analysis for a Spatio-Structural-Temporal Invasive Carcinoma Model
}

\author{
Arran Hodgkinson - Mark A. J. \\ Chaplain • Pia Domschke • Dumitru \\ Trucu
}

Received: 23 September 2017/ Accepted: date

\begin{abstract}
Spatio-temporal models have long been used to describe biological systems of cancer but it has not been until very recently that increased attention has been paid to structural dynamics of the interaction between cancer populations and the molecular mechanisms associated with local invasion. One system that is of particular interest is that of the urokinase plasminogen activator (uPA) wherein uPA binds uPA receptors (uPARs) on the cancer cell surface, allowing plasminogen to be cleaved into plasmin, which degrade the extra cellular matrix (ECM) and this way leads to enhanced cancer cell migration.

In this paper we develop a novel numerical approach and associated analysis for spatio-structuro-temporal modelling of the uPA system for up to twospatial and two-structural dimensions. This is accompanied by analytical exploration of the numerical techniques used in simulating this system, with special consideration being given to the proof of stability within numerical regimes encapsulating a central differences approach to approximating numerical gradients. The stability analysis performed here reveals instabilities induced by the coupling of the structural binding and proliferative processes. The numerical results expounds how the uPA system aids the tumour in in-

Arran Hodgkinson

DIMNP, Université de Montpellier II, Montpellier, 34095, France,

E-mail: arran.hodgkinson@etu.umontpellier.fr

Mark A. J. Chaplain

School of Mathematics and Statistics, Mathematical Institute, University of St. Andrews, St. Andrews, KY16 9SS, Scotland, UK

E-mail: majc@st-andrews.ac.uk

Pia Domschke

Technische Universität Darmstadt, Fachbereich Mathematik, Darmstadt, 64293, Germany

E-mail: domschke@mathematik.tu-darmstadt.de

Dumitru Trucu

Division of Mathematics, University of Dundee, Dundee, DD1 4HN, Scotland, UK

E-mail: trucu@maths.dundee.ac.uk
\end{abstract}


vading the local stroma, whilst the inhibitor to this system may impede this behaviour and encourage a more sporadic pattern of invasion.

Keywords Cancer Invasion - Structured Cell Population Dynamics . Computational Modelling

Mathematics Subject Classification (2000) AMS Subject Classification: $22 \mathrm{E} 46,53 \mathrm{C} 35,57 \mathrm{~S} 20$

\section{Introduction}

Over the past three decades or so, the processes involved in cancer growth and spread received significant mathematical attention through novel and increasingly sophisticated modelling approaches $[84,77,79,7,48]$, leading to a deeper understanding of key cancer development aspects with potential therapeutical importance $[42,13]$.

While being sometimes regarded as a paradigm of local tissue remodelling, cancer invasion is a crucially important process in the overall cancer development where complex heterotypic cell population processes combined with a cascade of molecular signalling mechanisms are leading to the degradation the healthy tissue and its concomitant repopulation by migratory cancer cells [30, 58]. This attracted a wide range of spatio-temporal modelling at either one spatial scale[7,50,6,20,21,22] or in a multiscale approach[96,80]. However, it became increasingly apparent that the context of macro-scale spatio-temporal modeling was not sufficient to take into account the intricate behaviour of cancer cell processes. To that end, with insights from important concepts in structural modelling of biological systems (considering age, size, etc.) $[37,66$, $78,26,38,10]$, the various spatio-temporal modelling approaches for cancer invasion have been recently complemented by structural models [24,41], which enable a more detailed description of the involved biological processes by implicitly accounting for a certain extent of single cell-dynamics.

One biological system, important in cancer invasion, that has received an increase attention in recent years has been termed the urokinase plasminogen activator ( $\mathrm{uPA})$ system $[80,89,82]$. The uPA protein has long been noted as a marker of various cancer types, such as colorectal, gastric, œsophageal, lung, cervical, ovarian, renal, pancreatic, and hepatocellular, with its greatest prognostic evidence being derived from strains of breast cancer [43]. The reduction in uPA expression in peritumoural tissue also causes this protein to be of great clinical significance, such that expression remains localised to the cancerous tissue $[15,70]$.

Urokinase plasminogen activator receptor (uPAR) is a protein anchored to the cell surface and, bound with high affinity to uPA [92], aids the degradation of the extra cellular matrix (ECM) [65,71]. X-ray analysis of the uPA-uPAR complex has revealed that $\mathrm{uPA}$ binds its receptor on a subsurface encapsulated by all three of its major interactive domains [60,9]. Neither uPAR nor unbound uPA are intrinsically active within the human tissue due to their folding being 
unfavourable to binding plasminogen, until formation of the uPA-uPAR complex [46]. However, regardless the biological paradigm, uPA retains its high specificity for plasminogen [86].

Further to this, the binding structure of these proteins allows the binding regions of the UPAR protein to become available for plasminogen protein interactions. Moreover, bound uPA is susceptible to being further bound by the class of inhibitory proteins referred to as uPA inhibitor-1 (uPAI-1). The ability of the cell to advantageously manipulate its environment and achieve local dominance, is further altered by this chemical adaptation. This process is mediated through changes to cellular capabilities when bound to activated uPA-uPAR complexes, enabling greater survival, adhesion, and migration [17]. It has been shown that even modest increases in the presence of this surfacebound complex are sufficient to greatly increase the prolific and proteolytic activities of invasive tumour cells [91].

The cancer invasive process is further augmented through integrin-mediated signalling pathways utilised by the uPA-uPAR complex [63]. Perhaps most significant is the activation of the protein class known as $\alpha 5 \beta 1$ integrin, which actively recruits the epidermal growth factor receptor (EGFR), an upstream signalling protein whose presence has been an indicator for high levels of extracellular signal-regulated kinases (ERKs) [72]. As an essential upregulator of mitotic activity in cancerous cells, ERKs enhance the proteolytic dynamics of the cancer cell population [25].

Furthermore, the complex formed between uPA and uPAR also increases the avidity of uPAR for vitronectin, an important protein for cell-ECM adhesion [99]. Vitronectin is a protein found primarily within plasma or deposited within the ECM, where it weakly binds the intra-matrix vitronectin receptor [100]. The unbound receptor, uPAR, will further selectively bind vitronectin and increase cellular adhesion to the ECM [98] whereas bound uPAR is an exceptionally high affinity receptor for vitronectin $[100,98]$.

Cancer cells migration is enhanced through the downstream synthesis of matrix metalloproteinases (MMPs), after the activation of conformal proMMP proteins by locally activated plasminogen, which degrade the ECM and enable local tumour invasion [30]. The growth of the tumour, however, then activates a negative feedback loop through the downstream upregulation of PAI-1 synthesis [52,69].

There is significant evidence that proteolytic enzymes (which degrade the collagen of the ECM) can be activated by an increased presence of activated plasminogen $[75,29]$. Primarily, the function of uPA is the conversion of plasminogen to plasmin; known to be a key regulator of these proteolytic proteins [29]. In this context, matrix metalloproteinase 2 (MMP2) is a major target for plasmin, causing increased degradation of the ECM and incorporation of the degraded collagen into localised plaques $[81,75]$.

Finally, a specifically prolific feature of the the uPA-uPAR complex (in relation to with its environment) is that in its active conversion of plasminogen to plasmin it encourages the production of the proenzyme single-chain urokinase plasminogen activator (scuPA). This scuPA protein is the precursor 
of uPA and therefore closes a positive feedback loop which is integral to the success of cancer cells in their invasive pursuit [17]. Plasmin is also capable of activating scuPA by cleaving a bond named Lys158 [86], contributing to the feedback mechanism. There is mounting evidence that the majority of these feedback mechanisms are localised to the cancer's invading edge $[99,30]$.

Based upon the biological evidence discussed so far, it is therefore crucially important to account for the molecular binding of the uPA components in modelling cancer dynamics. To that end, the general modelling approach introduced in Domschke et al.[41], where a novel spatio-temporal-structural model was derived for a general tissue dynamics involving of cells, ECM, and several accompanying populations of potentially membrane binding molecules, offers therefore an appropriate framework.

The model proposed by Domschke et al. [41] is a recent advancement within the well established area of a structured population modelling, uniquely utilising the structural dynamics to describe spatio-chemical-temporal processes in the tumour cell population. With a history stretching over almost six decades, however, structured-population models address a whole range of research challenges arising across many bio-medical and ecological areas, including epidemiology, collective movement either within cell population (such as those in cancer invasion or embryogenesis) or within social crowd dynamics. Varying in scope and purpose, these range from temporal-structural approaches (where space is ignored, and time is coupled for instance with "age" or "size" structure) $[93,94,88,55,37,66,56,57,97,36,38,62,19,87,27,10,24]$, to spatio-structural models were (where time is ignored) [53,74,39,49,61,85,67, $68,8,31,4]$, and finally to more complex approaches that couple time, space, and structure $[35,18,67,47,90,3,28,34,41,95]$. Specifically, important examples of structured approaches in cancer modelling include size-structured models [54] (which account for cell size in order to understand cell cycle dynamics), age-structured models $[14,44,45]$ (which account for the age distribution of the population), as well as more specific models taking into account various other aspects such as RNA content [64], mutational state [32,73,33], popular altruism [59] and more. For a more extensive introduction to and analysis of these structured cancer development models, one may refer to the review papers by Bellomo et al. $[12,11]$ as well as to a number of relevant books on this topic $[1,27,83,76]$.

Thus, adopting here the notations from Domschke et al.[41], in this paper we propose appropriate computational approaches and resulting simulation alongside associated analysis to explore the spatio-temporal-structural modelling of a cancerous tissue consisting of:

- a structured cancer cell density $c(t, x, y)$, with $(t, x, y) \in \mathcal{I} \times \mathcal{D} \times \mathcal{P}$, where $\mathcal{I}:=[0, T]$ is a time interval, $\mathcal{D} \in \mathbb{R}^{d}, d=1,2$, is the spatial tumour domain, and $\mathcal{P} \subset \mathbb{R}^{p}$ is a cone of appropriate dimension $p \leq 2$ representing the set of all admissible membrane-binding structures for the uPA System;

- ECM density $v(t, x)$, with $(t, x) \in \mathcal{I} \times \mathcal{D}$; 
- $q \leq 3$ components of the uPA system (uPA, PAI-1, and plasmin), which are appropriately grouped in binding and unbinding classes of molecular species represented here by $m_{b}:=\left[m_{b, 1}(t, x), \ldots, m_{b, p}(t, x)\right]^{T} \in \mathbb{R}^{p}$ and $m_{f}:=\left[m_{f, 1}(t, x), \ldots, m_{f, q-p}(t, x)\right]^{T} \in \mathbb{R}^{q-p}$, respectively.

\section{Structured Model for Cancer Modeling exploring the uPA binding dynamics}

Using on the theoretical framework derived in Domschke et al.[41], we explore the dynamics of a cancerous tissue of the resulting spatio-temporal-structural uPA modelling system, which we briefly describe here as follows. Per unit time, under the presence of a cell proliferation law, we generally assume that the spatial dynamics of the cancer cell population is dictated by diffusion, chemotaxis, haptotaxis, and cell-adhesion. The molecular binding and unbinding of the uPA components (uPA or PAI-1) is accounted for in this framework[41] in terms of an appropriately derived structural cone $\mathcal{P}$ (detailed on specific cases in the following sections), and the resulting dynamics leads not only to a spatio-temporal migration but also to a structural movement of the cancer population $c(t, x, y)$. The influence of cell-adhesion over the spatial dynamics at $x$ is considered here in non-structured fashion and, similar to other previous approaches[40,51], this is captured via a non-local term that represents the cell-cell and cell matrix adhesion interactions within a sensing region of radius $R$. This non-local term is of the form

$$
\mathcal{A}(t, x, y, \mathbf{u}(t, \cdot))=\frac{1}{R} \int_{B(0, R)} \mathbf{n}(\tilde{x}) \mathcal{K}\left(\|\tilde{x}\|_{2}\right) g(t, y, \mathbf{u}(t, x+\tilde{x})) \chi_{D}(x+\tilde{x}) d \tilde{x}
$$

where, for any $\tilde{x} \in B(0, R), \mathbf{n}(\tilde{x})$ represents the unit vector pointing from $x$ to $x+\tilde{x}, \mathcal{K}(\cdot)$ is a smooth spatial kernel, and the adhesion function $g(t, y, \mathbf{u}(t, x+$ $\tilde{x})$ ) accounts for the cell-cell and cell-matrix adhesion strengths $S_{c c}$ and $S_{c v}$, respectively, this being given by

$$
g(t, y, \mathbf{u})=\left[S_{c c}(t) \int_{\mathcal{P}} c d y+S_{c v}(t) v\right] \cdot(1-\rho(\mathbf{u}))^{+} .
$$

with the convenience vector notation

$$
\mathbf{u}(t, x):=\left[\int_{\mathcal{P}} c(t, x, y) d y, v(t, x)\right]^{T}
$$

and $(\cdot)^{+}:=\max \{0, \cdot\}$. Furthermore, as the ECM density $v(t, x)$ is only degraded and remodelled by the cancer cells and that the unbound (free) part of the considered components of the uPA System that are produced by the cancer cells are only diffusing in the tumour domain, the structured system 
that is obtained via the general modelling framework from Domschke et al.[41] is as follows:

$$
\left\{\begin{aligned}
\frac{\partial c}{\partial t}= & \nabla_{x} \cdot\left[D_{c} \nabla_{x} c-c(1-\rho(\mathbf{u}))\left(\sum_{k=1}^{q} \xi_{k} \nabla_{x} m_{k}+\xi_{v} \nabla_{x} v\right)+c \mathcal{A}\right] \\
& -\nabla_{y} \cdot[(\mathbf{b}(y, \mathbf{m})-\mathbf{d}(y)) c] \\
& +2^{p+1} \phi(2 y, c, v) c(t, x, 2 y)-\phi(y, c, v) c(t, x, y) \\
\frac{\partial v}{\partial t}= & -\gamma_{v}^{T} \mathbf{r} v+\psi_{v}(t, \mathbf{u}) \\
\frac{\partial \mathbf{m}}{\partial t}= & \nabla_{x} \cdot\left[\mathbf{D}_{\mathbf{m}} \nabla_{x} \mathbf{m}\right]-\int_{\mathcal{P}}(\hat{\mathbf{b}}(y, \mathbf{m})-\hat{\mathbf{d}}(y)) \varepsilon c(t, x, y) d y \\
& +\psi_{\mathbf{m}}(\mathbf{u}, \mathbf{r})-\operatorname{diag}\left(\gamma_{\mathbf{m}}\right) \mathbf{m} .
\end{aligned}\right.
$$

where the vector notations are used here to represent

- the molecular population of unbound (free) part of the considered components of uPA system via $\mathbf{m}:=\left[m_{b}^{T}, m_{f}^{T}\right]^{T}$;

- the total molecular population (both bound and unbound part) of the considered components of uPA system via $\mathbf{r}:=\left[\int_{\mathcal{P}} y \varepsilon c(t, x, y) d y, \mathbf{m}(t, x)\right]^{T}$, whereas $\varepsilon$ stands for the ratio between cell-surface density and cancer cell density. Furthermore, to simplify the context, the cell cancer proliferation law $\phi(y, c, v)$ is chosen here to be of a non-structured logistic form, namely

$$
\phi(y, \mathbf{u})=\mu_{c}(1-\rho(\mathbf{u}))
$$

where $\rho(\mathbf{u})$ quantifies the space occupied by the ECM and total cancer cell density

$$
C(t, x):=\int_{\mathcal{P}} c(t, x, y) d y
$$

and is defined by

$$
\rho(\mathbf{u}(t, x)):=\mathfrak{v}_{c} C(t, x)+\mathfrak{v}_{v} v(t, x)
$$

with $\mathfrak{v}_{c}$ and $\mathfrak{v}_{v}$ denoting the volume fraction for $c$ and $v$ at the same spatiotemporal point $(t, x)$, respectively. Moreover, the ECM remodelling term $\psi_{v}(t, \mathbf{u})$ assumes here the volume filling form

$$
\psi_{v}(t, \mathbf{u}):=\mu_{v}(1-\rho(\mathbf{u}))^{+} .
$$

Finally, for the uPA binding components given by $m_{b}:=\left[m_{b, 1}, \ldots, m_{b, p}\right]^{T}$, the cell surface binding and unbinding rates are represented here by $\mathbf{b}(y, \mathbf{m})$ and $\mathbf{d}(y)$, respectively. Therefore, since for the free components $m_{f}$ we do not have any binding or unbinding, to unify the notation, we use here the extended binding and unbinding rates vectors $\hat{\mathbf{b}}(y, \mathbf{m})$ and $\hat{\mathbf{d}}(y)$ in $\mathbb{R}^{q}$ given by

$$
\hat{\mathbf{b}}(y, \mathbf{m}):=\left[(\mathbf{b}(y, \mathbf{m}))^{T}, 0, \ldots, 0\right]^{T} \text { and } \hat{\mathbf{d}}(y):=\left[(\mathbf{d}(y))^{T}, 0, \ldots, 0\right]^{T} .
$$

The molecular source $\psi_{\mathbf{m}}$ is assumed to depend here only on $\mathbf{u}$ and the total molecular population $\mathbf{r}$ while the constant vector $\gamma_{\mathbf{m}} \in \mathbb{R}^{q}$ represents the natural degradation rate of $\mathbf{m}$. 
2.1 The dynamics of uPA System with and without PAI-1

Assuming that a total amount $M$ of uPAR receptors is uniformly distributed on the surface of each cancer cell, in the following we explore the form and dynamics of the spatio-temporal-structural system (3) when considering uPA binding and unbinding to uPAR both in the presence and in the absence of binding PAI-1 inhibitor molecules. These will result in different structural dimensionalities that will be addressed below as appropriate.

\subsection{1 uPA System in the absence of PAI-1}

The first case that we consider here accounts only for the uPA bounding and unbinding molecules while ignoring the presence of PAI-1. In this context, the total number of considered uPA System components is $q=2$, and consists of

- a binding molecular species (i.e., uPA) represented by $m_{b}(t, x):=m_{b, 1}(t, x)$;

- a free molecular species (i.e., plasmin) represented by $m_{f}(t, x):=m_{f, 1}(t, x)$.

The amount of those uPA molecules among $m_{b, 1}(t, x)$ that are exercising binding to the available uPAR receptors is denoted here with $y$, and represents the binding structure of the cancer cell population distributed at the spatio-temporal node $(x, t)$. Thus, under the assumption of a certain level of membrane-binding saturation, after eventual normalisation, the collection of all the binding structures $\mathcal{P}$ is given here by the interval $[0,1]$, so the dimension of $\mathcal{P}$ is in this case $p=1$. Furthermore, after a brief calculation[41], the $\mathrm{uPA}$ binding rate $\mathbf{b}(y, \mathbf{m})$ is given by

$$
\mathbf{b}(y, \mathbf{m})=(1-y) m_{b, 1},
$$

while the $\mathrm{uPA}$ unbinding rate $\mathbf{d}(y)$ has a form

$$
\mathbf{d}(y)=d_{m_{b, 1}} .
$$

Furthermore, assuming that the unbound uPA is produced by the cancer cells $C(t, x)$ at the rate $\alpha_{m_{b, 1}}$ and that plasmin is produced only by those cells which are bound by uPA at a rate $\alpha_{m_{f, 1}}$, the molecular source term $\psi_{\mathbf{m}}(C, \mathbf{r})$ is given here by

$$
\psi_{\mathbf{m}}(C, \mathbf{r})=\left[\begin{array}{c}
\alpha_{m_{b, 1}} C \\
\alpha_{m_{f, 1}} \int_{\mathcal{P}} y \varepsilon c(t, x, y) d y
\end{array}\right]
$$

\subsection{2 uPA System in the presence of PAI-1}

Building on the first modelling case assumed in subsection 2.1.1, we consider now a second situation in which, besides the binding uPA, also the inhibitor PAI-1 is brought into the picture, this being able to bind to cell surface-bound uPA molecules, this way inhibiting their action. In this new context, the total number of the uPA System considered is $q=3$, this consisting of 
- two binding molecular species (i.e., uPA and PAI-1) represented by $m_{b}(t, x):=$ $\left[m_{b, 1}(t, x), m_{b, 2}(t, x)\right]^{T}$, with $m_{b, 1}(t, x)$ standing for the uPA density and $m_{b, 2}(t, x)$ denoting the PAI-1 inhibitor density;

- a free molecular species (i.e., plasmin) represented by $m_{f}(t, x):=m_{f, 1}(t, x)$.

While proceeding as in subsection 2.1.1 and denoting amount of those uPA molecules among $m_{b, 1}(t, x)$ that are exercising binding to the available uPAR receptors is denoted here with $y_{1}$, we denote with $y_{2}$ the amount of PAI1 receptors that binds to bound $\mathrm{uPA}$, causing the inhibition of these uPA molecules. Thus, given the binging possibilities for PAI-1 onto receptor-bound $\mathrm{uPA}$ versus the binding possibilities of the free $\mathrm{UPA}$ on the uPAR receptors, it is always the case that $y_{2} \leq y_{1}$, and so after an eventual normalisation due to reaching saturation levels of cell-surface uPA binding, we obtain that that maximal set of binding structures $\mathcal{P}$ is two-dimensional in this case and is given by $\mathcal{P}:=\left\{\left(y_{1}, y_{2}\right) \in \mathbb{R}^{2} \mid y_{1} \in[0,1]\right.$ and $\left.y_{2} \in\left[0, y_{1}\right]\right\}$. Thus, using a measure theoretical argument[41] for the binding components for the uPA and PAI-1, the vector of binding rates $\mathbf{b}(y, \mathbf{m})$ is given by

$$
\mathbf{b}(y, \mathbf{m})=\left[\begin{array}{c}
\left(1-y_{1}\right) \beta_{1} m_{b, 1} \\
\left(y_{1}-y_{2}\right) \beta_{2} m_{b, 2}
\end{array}\right]
$$

and similarly, we obtain that the vector of unbinding rates $\mathbf{d}(y)$ is

$$
\mathbf{d}(y)=\left[\begin{array}{c}
\left(y_{1}-y_{2}\right) d_{y_{1}} \\
y_{2} d_{y_{2}}
\end{array}\right]
$$

Assuming that the uPA density $m_{b, 1}$ is produced in the presence of cells expressing uPAR (namely the total cell density $C$ ) at a rate $\alpha_{m_{b, 1}}, m_{b, 2}$ is produced in the presence of activated plasminogen (namely the plasmin density $\left.m_{f, 1}\right)$ at a rate $\alpha_{m_{b, 2}}$, and plasmin density $m_{f, 1}$ is activated by cells expressing uPA density $m_{b, 1}$ but not also inhibitor PAI-1 density $m_{b, 2}$ at a rate $\alpha_{m_{f, 1}}$, we obtain that the molecular source term $\psi_{\mathbf{m}}$ is given by

$$
\psi_{\mathbf{m}}(C, \mathbf{r})=\left[\begin{array}{c}
\alpha_{m_{b, 1}} C \\
\alpha_{m_{b, 2}} m_{f, 1} \\
\alpha_{m_{f, 1}} \int_{\mathcal{P}}\left(y_{1}-y_{2}\right) \varepsilon c(t, x, y) d y
\end{array}\right] .
$$

\section{Computational Approach and Analysis of the Discretisation of the Spatio-Structural-Temporal Tumour Model}

Throughout this section, we consider only the case of one dimension both in space and structure for system (3). Thus, assuming equal spatial and structural step size $\delta_{x}=\delta_{y}$ and an equal number $r \in \mathbb{N} \backslash\{0,1,2,3\}$ of collocation points in both $x$ and $y$ dimensions, in the following we will proceed to discretise $c, v$, and $\mathbf{m}$ at any given time node $n \delta_{t}$, with $n \in \mathbb{N}$. At each discretised 
spatial location in $x$, let $c_{x}^{n}$ denote the vector of the discretisation of the distribution of the cancer cell population over the structural dimension $y$, explicitly given by $c_{x}^{n}:=\left[c_{x, y_{1}}^{n}, \ldots, c_{x, y_{r}}^{n}\right]^{T}$. Likewise, at each discretised structural location in $y$, let $c_{y}^{n}$ denote the vector of the discretisation of the distribution of the cancer cell population over the spatial dimension $x$, explicitly given by $c_{y}^{n}:=\left[c_{x_{1}, y}^{n}, \ldots, c_{x_{r}, y}^{n}\right]^{T}$. In a similar way, the spatial discretisation of the ECM concentration is denoted by $v^{n}:=\left[v_{x_{1}}^{n}, \ldots, v_{x_{r}}^{n}\right]^{T}$. Further, to simplify the notation for the components of $\mathbf{m}$, in this section we will drop the indices $b$ and $f$ and orderly relabel the involved molecular species simply upon their position in the vector $\mathbf{m}$, namely as $\mathbf{m}=\left[m_{1}, \ldots, m_{q}\right]^{T}$. In this context, the discretisation of $\mathbf{m}$ is simply denoted by $\mathbf{m}^{n}:=\left[\left(m_{1}^{n}\right)^{T}, \ldots,\left(m_{q}^{n}\right)^{T}\right]^{T} \in \mathbb{R}^{q r}$, with $m_{i}^{n}:=\left[m_{i, x_{1}}^{n}, \ldots, m_{i, x_{r}}^{n}\right]^{T}, \forall i \in\{1, \ldots, q\}$.

Finally, for appropriately designed $r \times r$ diagonal matrices $\Gamma$ (aimed to serve for approximating expectations of the various structurally distributed variables that are involved in system (3)), let us denote $C^{n}(\Gamma):=\left[C_{x_{1}}^{n}(\Gamma), \ldots, C_{x_{r}}^{r}(\Gamma)\right]^{T} \in$ $\mathbb{R}^{r}$, with each component defined by

$$
C_{x_{i}}^{n}(\Gamma):=\frac{\delta_{y}}{2}\left[c_{x_{i}}^{n}\right]^{T} \Gamma[1,2,2, \ldots, 2,2,1]^{T},
$$

and note that for instance the total cell density is given by $C\left(I_{r}\right)$, where $I_{r}$ is the $r \times r$ identity matrix.

\subsection{Discretisation of the 1D-Spatial 1D-Structural uPA Model}

The iterative time step for the cancer population equation in (3) is then given by

$$
c^{n+1}=c^{n}+\delta_{c}^{n} \text { with } \delta_{c}^{n}=\left(A_{x} c_{x}^{n}+\left(A_{y}+A_{\phi}\right) c_{y}^{n}\right) \cdot \delta_{t}
$$

where

$$
\begin{aligned}
A_{x} & =\frac{1}{4 \delta_{x}^{2}} J^{2}+\frac{1}{2 \delta_{x}} J \tilde{f} \\
\tilde{f} & :=\operatorname{diag}\left(\left[f_{1}, \ldots, f_{r}\right]\right),
\end{aligned}
$$

with $J_{r}$ being the $r \times r$ central difference derivative matrix given by

$$
J_{r}=\left(\begin{array}{ccccccc}
-2 & 2 & 0 & \ldots & 0 & 0 & 0 \\
-1 & 0 & 1 & \ldots & 0 & 0 & 0 \\
0 & -1 & 0 & \ddots & 0 & 0 & 0 \\
\vdots & \vdots & \ddots & \ddots & \ddots & \vdots & \vdots \\
0 & 0 & 0 & \ddots & 0 & 1 & 0 \\
0 & 0 & 0 & \ldots & -1 & 0 & 1 \\
0 & 0 & 0 & \ldots & 0 & -2 & 2
\end{array}\right),
$$


and the components of $\tilde{f}$ being given by

$$
f_{i}:=f_{i}\left(c_{x_{i}}^{n}, v_{x_{i}}^{n}, \mathbf{m}_{x_{i}}^{n}\right)=\left(1-\rho\left(c_{x_{i}}^{n}, v_{x_{i}}^{n}\right)\left(\sum_{k} \xi_{k} \operatorname{row}_{i}\left(J_{r}^{2}\right) \mathbf{m}_{k, x_{i}}^{n}+\xi_{v} \operatorname{row}_{i}\left(J_{r}^{2}\right) v_{x_{i}}^{n}\right),\right.
$$

where $\operatorname{row}_{i}\left(J_{r}^{2}\right)$ indicates the $i^{\text {th }}$ row of the matrix given by $J_{r}^{2}$, for all $i \in$ $\{1, \ldots, r\}$. Furthermore, we have that

$$
\begin{aligned}
A_{y} & =-\frac{1}{2 \delta_{y}} J_{r} \tilde{g} \\
\tilde{g} & :=\operatorname{diag}\left(\left[\left(b_{1}-d_{1}\right), \ldots,\left(b_{r}-d_{r}\right)\right]\right),
\end{aligned}
$$

where $b_{i}:=b\left(\mathbf{m}^{n}, y_{i}\right)$ and $d_{i}:=d\left(y_{i}\right), \forall i \in\{1, \ldots, r\}$ stands for the discretised binding and unbinding rates, and

$$
A_{\phi}=A_{\phi_{y}}+A_{\phi_{2 y}}
$$

with

$$
\begin{array}{rlrl}
A_{\overline{\phi_{y}}} & =-\tilde{\phi}_{y} & A_{\overline{\phi_{2 y}}}=2^{P+1} \hat{I}^{T} \tilde{\phi}_{2 y} \hat{I} \\
\tilde{\phi}_{y}=\operatorname{diag}\left(\left[\phi_{1}, \ldots, \phi_{r}\right]\right) & \tilde{\phi}_{2 y}=\sum_{i=1}^{r} \phi_{2 i} E_{i, 2 i} \quad \hat{I}=\left(\begin{array}{c}
I_{r} \\
\emptyset_{r}
\end{array}\right),
\end{array}
$$

where $\emptyset_{r}$ is the $r \times r$ zero matrix, $E_{i, 2 i}$ is the standard elementary matrix; $\phi_{i}:=\phi\left(y_{i}, c_{y_{i}}^{n}, v^{n}\right)$ and $\phi_{2 i}:=\phi\left(2 y_{i}, c_{2 y_{i}}^{n}, v^{n}\right), \forall i \in\{1, \ldots, r\}$.

Similarly iterative time step for the ECM equation in (3) is given by

$$
v^{n+1}=v^{n}+\delta_{v}^{n} \text { with } \delta_{v}^{n}=\left(B_{x} v^{n}+\tilde{\psi}_{v}\right) \cdot \delta_{t},
$$

where

$$
B_{x}=-\gamma_{v, c} \varepsilon \operatorname{diag}\left(C^{n}(\tilde{y})\right)-\sum_{i=1}^{q} \gamma_{v, m_{i}} \operatorname{diag}\left(m_{i}^{n}\right),
$$

with $\tilde{y}:=\operatorname{diag}\left(\left[y_{1}, \ldots, y_{r}\right]\right)$, the ECM degradation rates vector $\gamma_{v}$ organised as $\gamma_{v}:=\left[\gamma_{v, c}, \gamma_{v, m_{1}}, \ldots, \gamma_{v, m_{q}}\right]^{T}$. Furthermore, $\tilde{\psi}_{v}$ denotes here the remodelling vector given by

$$
\tilde{\psi}_{v}:=\left[\psi_{v, 1}, \ldots, \psi_{v, r}\right]^{T},
$$

where we use the reduced notation $\psi_{v, i}:=\psi_{v}\left(c_{i}^{n}, v_{i}^{n}\right), \forall i \in\{1, \ldots, r\}$.

Finally, the iterative time step for uPA components equation in (3) is given by

$$
\mathbf{m}^{n+1}=\mathbf{m}^{n}+\delta_{\mathbf{m}}^{n} \text { with } \delta_{\mathbf{m}}^{n}=\left(D_{x}\left(\mathbf{m}^{n}\right)+D_{\phi}\right) \cdot \delta_{t},
$$

where we used the operator notation $D_{x}\left(\mathbf{m}^{n}\right):=\left[\left(D_{m_{1}} J_{r}^{2} m_{1}^{n}\right)^{T}, \ldots,\left(D_{m_{q}} J_{r}^{2} m_{q}^{n}\right)^{T}\right]^{T}$, and

$$
D_{\phi_{i}}:=\psi_{m_{i}}-\varepsilon C^{n}(\tilde{g})-\gamma_{m_{i}} m_{i}^{n}
$$

where $\psi_{m_{i}}:=\left[\psi_{m_{i}}\left(C_{x_{1}}^{n}\left(I_{r}\right), C_{x_{1}}^{n}(\tilde{y})\right), \ldots, \psi_{m_{i}}\left(C_{x_{r}}^{n}\left(I_{r}\right), C_{x_{r}}^{n}(\tilde{y})\right)\right]^{T}, \forall i \in\{1, \ldots, q\}$, where we used the operator notation $D_{\phi}\left(\mathbf{m}^{n}\right):=\left[D_{\phi_{1}}^{T}, \ldots, D_{\phi_{q}}^{T}\right]^{T}$. 
Therefore, the first iteration of the resulting discrete global operator for an arbitrary discrete spatio-structural points $(x, y)$, and time node $n=0$, which appears when computing given by $\left(c^{1}, v^{1}, \mathbf{m}^{1}\right)^{T}$, leads to the following relations:

$$
\begin{aligned}
\delta_{c}^{0}= & \left(\frac{1}{4 \delta_{x}^{2}} J_{r}^{2} c_{y}^{0}+\frac{1}{2 \delta_{x}} J_{r} \tilde{f} c_{y}^{0}-\frac{1}{2 \delta_{y}} J_{r} \tilde{g} c_{x}^{0}\right. \\
& \left.+\left(2^{P+1} \hat{I}^{T} \tilde{\phi}_{2 y} \hat{I}-\tilde{\phi}_{y}\right) c_{y}^{0}\right) \delta_{t} \\
\delta_{v}^{0}= & \left(-\gamma_{v, c} \varepsilon \operatorname{diag}\left(C^{n}(\tilde{y})\right) v^{0}-\sum_{k=1}^{q} \gamma_{v, m_{k}} \operatorname{diag}\left(m_{k}^{n}\right) v^{0}+\tilde{\psi}_{v}\right) \delta_{t} \\
\delta_{m_{k}}^{0}= & \left(D_{m_{k}} J_{r}^{2} m_{k}^{n}+\psi_{m_{k}}-\varepsilon C^{0}(\tilde{g})-\gamma_{m_{k}} m_{k}^{n}\right) \delta_{t} .
\end{aligned}
$$

The stability of the primary term in these discrete time differences is dependent upon the operator $J_{r}$ whose analysis will be the focus of the following subsection.

3.2 Stability analysis considerations on the central difference operator $J_{r}$

In order to assess the stability of the difference operator $J_{r}$ involved in (43), in the following we will prove a series of technical results that will ultimately completely characterise the eigenvalues of $J_{r}$.

Lemma 1 Let $\mathcal{Q}$ be the following set of polynomials with real coefficients

$$
\mathcal{Q}:=\left\{\begin{array}{l|l}
P_{k}(x)=a_{k} x^{k}+\cdots+a_{1} x+a_{0} & \begin{array}{l}
k \geq 4, \\
a_{k-1-2 i}=0, \forall i \in 0, \ldots,\left[\frac{k-1}{2}\right]
\end{array}
\end{array}\right.
$$

where by [.] we understand the usual integer part. Further, let $P_{N-2}, P_{N-1} \in \mathcal{Q}$ be polynomials of degree $N-2$ and $N-1$ respectively, then the iterative relation $P_{N}=P_{N-2}-x P_{N-1}$ gives rise to a polynomial of degree $N$ with $P_{N} \in \mathcal{Q}$.

Proof If one writes the considered polynomials as

$$
\begin{gathered}
P_{N-2}: a_{N-2} x^{N-2}+a_{N-4} x^{N-4}+\ldots+\max _{i \in\left\{0, \ldots,\left[\frac{k-1}{2}\right]\right\}} a_{N-i-3} x^{N-i-3}=0 \\
P_{N-1}: a_{N-1} x^{N-1}+a_{N-3} x^{N-3}+\ldots+\max _{i \in\left\{0, \ldots,\left[\frac{k-1}{2}\right]\right\}} a_{N-i-2} x^{N-i-2}=0,
\end{gathered}
$$

then the proof is trivial.

Theorem 1 Considering the set of polynomials $\mathcal{Q}$ defined in (28), for any natural $N \geq 6$, let $P_{N} \in \mathcal{Q}$ be a polynomial of degree $N$ such that the polynomials $P_{N-2}, P_{N-1} \in \mathcal{Q}$ that give $P_{N}$ via the recurrence relation $P_{N}=$ $P_{N-2}-x P_{N-1}$ satisfy the following properties: 
1) denote $\left\{u_{1}, u_{2}, \ldots, u_{N-2}\right\}$ and $\left\{v_{1}, v_{2}, \ldots, v_{N-1}\right\}$ the ordered set of roots of the polynomials $P_{N-2}$ and $P_{N-1}$, respectively

2) the roots of these two polynomials are only imaginary, namely:

$$
\begin{array}{ll}
\operatorname{Re}\left(u_{i}\right)=0 & \forall i \in\{1, \ldots, N-2\} \\
\operatorname{Re}\left(v_{j}\right)=0 & \forall j \in\{1, \ldots, N-1\}
\end{array}
$$

3) finally, the roots of these two polynomials satisfy the additional relations:

$$
\text { and } \begin{aligned}
& v_{N-1}^{2} \geq u_{N-2}^{2} \\
& \quad u_{i}^{2} \geq v_{i}^{2} \geq u_{i-2}^{2} \geq v_{i-2}^{2}, \quad \forall i \in\left\{i=2 j \mid j \in\left\{1, \ldots,\left[\frac{N-2}{2}\right]\right\}\right\},
\end{aligned}
$$

where for any $i \in\left\{i=2 j \mid j \in\left\{1, \ldots,\left[\frac{N-2}{2}\right]\right\}\right\}$ we have that $u_{j-1}:=\bar{u}_{j}$ and $v_{j-1}:=\bar{v}_{j}$.

Then, if we let $\left\{w_{1}, w_{2}, \ldots, w_{N}\right\}$ denote the ordered set of roots for $P_{N}$, we have that

i) all the roots of $P_{N}$ are imaginary, i.e.,

$$
\operatorname{Re}\left(w_{i}\right)=0, \quad \forall i \in\{1 . . N\},
$$

ii) the roots of $P_{N}$ and $P_{N-1}$ satisfy the relations:

$$
\text { and } \begin{aligned}
& w_{N-1}^{2} \geq v_{N-2}^{2} \\
& \quad v_{i}^{2} \geq w_{i}^{2} \geq v_{i-2}^{2} \geq w_{i-2}^{2}, \quad \forall i \in\left\{i=2 j \mid j \in\left\{1, \ldots,\left[\frac{N-1}{2}\right]\right\}\right\},
\end{aligned}
$$

where for any $i \in\left\{i=2 j \mid j \in\left\{1, \ldots,\left[\frac{N-1}{2}\right]\right\}\right\}$ we have that $w_{j-1}:=\bar{w}_{j}$.

Proof First, we notice that there are 2 different cases:

(1) $N$ is even or

(2) $N$ is odd.

Take case (1) and let $N=2 n, n \in \mathbb{N}$. We can use the conjugate root theorem to write the polynomials as

$$
\begin{aligned}
& P_{N-2}(x)=\left(x^{2}-u_{2}^{2}\right)\left(x^{2}-u_{4}^{2}\right) \ldots\left(x^{2}-u_{N-2}^{2}\right) \\
& P_{N-1}(x)=-x\left(x^{2}-v_{2}^{2}\right)\left(x^{2}-v_{4}^{2}\right) \ldots\left(x^{2}-v_{N-2}^{2}\right),
\end{aligned}
$$

where $u_{2 i-1}=\overline{u_{2 i}}, u_{2 i}^{2}=u_{2 i-1} \cdot u_{2 i}$ and $v_{2 i-1}=\overline{v_{2 i}}, v_{2 i}^{2}=-\left|\operatorname{Im}\left(v_{2 i}\right)^{2}\right|$. We then have that $P_{N}$ can be written as

$P_{N}(x)=\left(x^{2}-u_{2}^{2}\right)\left(x^{2}-u_{4}^{2}\right) \ldots\left(x^{2}-u_{N-2}^{2}\right)+x^{2}\left(x^{2}-v_{2}^{2}\right)\left(x^{2}-v_{4}^{2}\right) \ldots\left(x^{2}-v_{N-2}^{2}\right)$.

Now, by Descartes' rule of signs and Lemma 1, we can say that none of the roots of $P_{N}$ are positive and that at most $N$ of these roots are negative. 
Use the substitution $z=x^{2}$, the initial polynomial $P_{N}$ induces

$$
\bar{P}_{N}(z)=\left(z-u_{2}^{2}\right)\left(z-u_{4}^{2}\right) \ldots\left(z-u_{N-2}^{2}\right)+z\left(z-v_{2}^{2}\right)\left(z-v_{4}^{2}\right) \ldots\left(z-v_{N-2}^{2}\right) .
$$

Evaluating $\bar{P}_{N}(z)$ at $\pm \infty$ and at a selection roots of $P_{N-1}(x)$ given by

$$
S_{a}:=\left\{v_{2 j}^{2} \mid j=1, n-1\right\},
$$

we have two different cases:

(1a) $n-1$ is odd or

(1b) $n-1$ is even.

In case (1a), we have that

$$
\lim _{z \rightarrow \pm \infty} \bar{P}_{N}(z)=+\infty .
$$

Further, as the elements of $S_{a}$ are solutions of $P_{N-1}$, using their properties that $u_{2 j}^{2} \geq v_{2 j}^{2}, j=1, n$, we obtain

$$
\bar{P}_{N}\left(v_{2 j}^{2}\right)=(-1)^{j}\left|v_{i}^{2}-u_{2}^{2}\right|\left|v_{i}^{2}-u_{4}^{2}\right| \ldots\left|v_{i}^{2}-u_{N-2}\right|, \quad j=1, n-1,
$$

and so

$$
\operatorname{sign}\left(\bar{P}_{N}\left(v_{2 j}^{2}\right)\right)=(-1)^{j}, \quad j=1, n-1
$$

Therefore, denoting $S_{a}^{\prime}:=\{-\infty\} \cup S_{a} \cup\{+\infty\}$, we have

$$
\operatorname{sign}\left(\bar{P}_{N}\left(S_{a}^{\prime}\right)\right)=\{+,-,+,-, \ldots,-,+\},
$$

which yields $n$ intervals where the values of polynomial changes, and so by Intermediate Value Theorem we must have $n$ real non-positive roots for $\bar{P}_{N}(z)$. Thus, reversing now the change of variable $z=x^{2}$, we obtain that the initial polynomial $P_{N}(x)$ has only imaginary roots with

$$
\operatorname{Re}\left(w_{i}\right)=0 \quad \text { with } \quad w_{i} \geq v_{i}, \quad i=1, N
$$

For case (1b), we have that

$$
\lim _{z \rightarrow \pm \infty} \bar{P}_{N}(z)= \pm \infty
$$

Further, denoting by with $S_{b}$ the following set of squares of the roots of $P_{N-2}(x)$, namely

$$
\begin{gathered}
S_{b}:=\left\{u_{2 j}^{2} \mid j=1, n-1\right\}, \\
\operatorname{sign}\left(\bar{P}_{N}\left(u_{2 j}^{2}\right)\right)=(-1)^{n-j}, \quad j=1, n-1
\end{gathered}
$$

Therefore, denoting $S_{b}^{\prime}:=\{-\infty\} \cup S_{b} \cup\{+\infty\}$, we have

$$
\operatorname{sign}\left(\bar{P}_{N}\left(S_{b}^{\prime}\right)\right)=\{-,+,-,+, \ldots,-,+\},
$$

and by the Intermediate Value Theorem, we again get $\operatorname{Re}\left(w_{i}\right)=0$ with $w_{i} \geq v_{i}$. 
For case (2), we consider odd values of $N$ and let $N=2 n+1, n \in \mathbb{N}$ such that we again use the conjugate root theorem to write

$$
\begin{aligned}
P_{N-2}(x) & =-x\left(x^{2}-v_{2}^{2}\right)\left(x^{2}-v_{4}^{2}\right) \ldots\left(x^{2}-v_{N-2}^{2}\right) \\
P_{N-1}(x) & =\left(x^{2}-u_{2}^{2}\right)\left(x^{2}-u_{4}^{2}\right) \ldots\left(x^{2}-u_{N}^{2}\right) \\
P_{N}(x) & =-x\left(x^{2}-u_{2}^{2}\right)\left(x^{2}-u_{4}^{2}\right) \ldots\left(x^{2}-u_{n-2}^{2}\right)-x\left(x^{2}-v_{2}^{2}\right)\left(x^{2}-v_{4}^{2}\right) \ldots\left(x^{2}-v_{N}^{2}\right) .
\end{aligned}
$$

Further, factoring out the common multiple $-x$ and using the substitution $z=x^{2}$ to augment the remainder of the polynomial, we can now test the polynomial with the set, $S^{\prime}$, in the same way as in case (1).

Theorem 2 Assuming that $N$ is the number of discretisation points and $N \geq$ 6 , the characteristic polynomials $P_{N}(\lambda)$ of the central differences matrices $J_{N}$ satisfy the following recurrence relation

$$
P_{N}(\lambda)=P_{N-2}(\lambda)-\lambda P_{N-1}(\lambda) .
$$

Proof Let's denote $P_{N}$ is the characteristic polynomial of the $N \times N$ dimensional central differences matrix, $J_{N}$, we observe first that desired the recurrence relation $P_{N}=P_{N-2}-\lambda P_{N-1}$ is trivially satisfied by the characteristic polynomials of $J_{4}, J_{5}$, and $J_{6}$, which are given by

$$
\begin{aligned}
& P_{4}=\lambda^{4}+\lambda^{2} \\
& P_{5}=-\lambda^{5}-2 \lambda^{3} \\
& P_{6}=\lambda^{6}+3 \lambda^{4}+\lambda^{2} .
\end{aligned}
$$

To prove that this relation is satisfied in general for any natural number $N \geq 6$, we proceed as follows. First, $\forall l \in \mathbb{N} \backslash\{0,1,2,3\}$, for the matrix $\hat{J}_{l}:=J_{l}-\lambda I_{l}$, and let us denote by $A_{l}^{o}$ and $A_{l}^{\prime}$ the following determinants of the $(l-1) \times(l-1)$ submatrices of $\hat{J}_{l}$, namely

$$
\begin{aligned}
A_{l-1}^{o}= & \left|\begin{array}{ccccc}
\hat{J}_{2,2} & \hat{J}_{2,3} & \hat{J}_{2,4} & \ldots & \hat{J}_{2, l} \\
\hat{J}_{3,2} & \hat{J}_{3,3} & \hat{J}_{3,4} & \ldots & \hat{J}_{3, l} \\
\hat{J}_{4,2} & \hat{J}_{4,3} & \hat{J}_{4,4} & \ldots & \hat{J}_{4, l} \\
\vdots & \vdots & \vdots & \ldots & \vdots \\
\hat{J}_{l, 2} & \hat{J}_{l, 3} & \hat{J}_{l, 4} & \ldots & \hat{J}_{l, l}
\end{array}\right|, \\
A_{l-1}^{\prime}= & \left|\begin{array}{ccccc}
\hat{J}_{2,1} & \hat{J}_{2,3} & \hat{J}_{2,4} & \ldots & \hat{J}_{2, l} \\
\hat{J}_{3,1} & \hat{J}_{3,3} & \hat{J}_{3,4} & \ldots & \hat{J}_{3, l} \\
\hat{J}_{4,1} & \hat{J}_{4,3} & \hat{J}_{4,4} & \ldots & \hat{J}_{4, l} \\
\vdots & \vdots & \vdots & \ldots & \vdots \\
\hat{J}_{l, 1} & \hat{J}_{l, 3} & \hat{J}_{l, 4} & \ldots & \hat{J}_{l, l}
\end{array}\right|,
\end{aligned}
$$


and let's observe that these have the properties that

$$
\begin{aligned}
& A_{l-1}^{o}=-\lambda A_{l-2}^{o}-A_{l-2}^{\prime} \\
& A_{i-1}^{\prime}=-A_{l-2}^{o}
\end{aligned}
$$

Then, using (39) we have that

$$
\begin{aligned}
\left|\hat{J}_{N-2}\right| & =(-2-\lambda) A_{N-3}^{o}-2 A_{N-3}^{\prime} \\
\left|\hat{J}_{N-1}\right| & =(-2-\lambda) A_{N-2}^{o}-2 A_{N-2}^{\prime} \\
& =(-2-\lambda)\left(-\lambda A_{N-3}^{o}-A_{N-3}^{\prime}\right)-2\left(-A_{N-3}^{o}\right) \\
& =(-2-\lambda)(-\lambda) A_{N-3}^{o}+(2+\lambda) A_{N-3}^{\prime}+2 A_{N-3}^{o} \\
& =((-2-\lambda)(-\lambda)+2) A_{N-3}^{o}+(2+\lambda) A_{N-3}^{\prime} \\
\left|\hat{J}_{N}\right| & =(-2-\lambda) A_{N-1}^{o}-2 A_{N-1}^{\prime} \\
& =(-2-\lambda)\left(-\lambda A_{N-2}^{o}-A_{N-2}^{\prime}\right)-2\left(-A_{N-2}^{o}\right) \\
& =(-2-\lambda)\left(-\lambda\left(-\lambda A_{N-3}^{o}-A_{N-3}^{\prime}\right)-\left(-A_{N-3}^{o}\right)\right)+2 A_{N-2}^{o} \\
& =(-2-\lambda)\left(-\lambda\left(-\lambda A_{N-3}^{o}-A_{N-3}^{\prime}\right)-\left(-A_{N-3}^{o}\right)\right)+2\left(-\lambda A_{N-3}^{o}-A_{N-3}^{\prime}\right) \\
& =(-2-\lambda)(-\lambda)^{2} A_{N-3}^{o}+(-2-\lambda)(-\lambda) A_{N-3}^{\prime}+(-2-\lambda) A_{N-3}^{o}-2 \lambda A_{N-3}^{o}-2 A_{N-3}^{\prime} \\
& =\left((-2-\lambda)(-\lambda)^{2}+(-2-\lambda)-2 \lambda\right) A_{N-3}^{o}+((-2-\lambda)(-\lambda)-2) A_{N-3}^{\prime}
\end{aligned}
$$

From (40a)-(40c) we obtain immediately by direct calculation that

$$
\left|\hat{J}_{N}\right|=\left|\hat{J}_{N-2}\right|-\lambda\left|\hat{J}_{N-1}\right|
$$

which can finally be equivalently expressed as

$$
P_{N}(\lambda)=P_{N-2}(\lambda)-\lambda P_{N-1}(\lambda) .
$$

Therefore, we finally obtain the following central result for our analysis.

Theorem 3 The eigenvalues of the central differences matrix $J_{r}$ are either 0 or imaginary.

Proof Using Theorem 2 we have that the characteristic polynomial of $J_{r}$ is given by $P_{r}(\lambda)$ and that $u_{i}^{2} \geq v_{i}^{2} \geq u_{i-2}^{2} \geq v_{i-2}^{2}$ for all $i \in\{i=2 j \mid j \in$ $\left.\left\{1, \ldots,\left[\frac{r-2}{2}\right]\right\}\right\}$. Then, by invoking Theorem 1 , we immediately obtain by induction that the roots, denoted $w_{i}$, of characteristic polynomial of $J_{r}$ are imaginary with $\operatorname{Re}\left(w_{k}\right)=0, \forall k \in\{1, \ldots, r\}$. 
3.3 Stability analysis of the global numerical scheme

Returning now to the stability analysis of the global numerical scheme associated to 1D-spatio 1D-structural case of (3), we will focus now only those operators occurring in (43) that have eigenvalues $\operatorname{Re}(\lambda)>0$. Therefore, as $J_{r}$ was proved to be stable, of interest for the stability analysis remains the behaviour of the following remainders of the operators from (43) without those terms involving $J_{r}$ that we indicate with $^{-}$, namely:

$$
\begin{aligned}
\bar{\delta}_{c}^{0} & =\left(2^{P+1} \hat{I}^{T} \tilde{\phi}_{2 y} \hat{I}-\tilde{\phi}_{y}\right) c_{y}^{0} \delta_{t} \\
\bar{\delta}_{v}^{0} & =\left(-\gamma_{v, c} \varepsilon \operatorname{diag}\left(C^{n}(\tilde{y})\right) v^{0}-\sum_{k=1}^{q} \gamma_{v, m_{k}} \operatorname{diag}\left(m_{k}^{n}\right) v^{0}+\tilde{\psi}_{v}\right) \delta_{t} \\
\bar{\delta}_{m_{k}}^{0} & =\left(\psi_{m_{k}}-\varepsilon C^{0}(\tilde{g})-\gamma_{m_{k}} m_{k}^{n}\right) \delta_{t} .
\end{aligned}
$$

We begin by assessing the stability of the structural dimension by considering the mitotic operator for the $2^{\text {nd }}$ and $3^{\text {rd }}$ such iterations on $c$, wherein we have:

$$
\begin{aligned}
\bar{\delta}_{c}^{1}= & \left(A_{\overline{\phi_{y}}}+A_{\overline{\phi_{2 y}}}\right) c_{y}^{0} \delta_{t}+\left(A_{\overline{\phi_{y}}}+A_{\overline{\phi_{2 y}}}\right)^{2} c_{y}^{0} \delta_{t}^{2}, \\
\bar{\delta}_{c}^{2}= & \left(A_{\overline{\phi_{y}}}+A_{\overline{\phi_{2 y}}}\right) c_{y}^{0} \delta_{t}+\left(A_{\overline{\phi_{y}}}+A_{\overline{\phi_{2 y}}}\right)^{2} c_{y}^{0} \delta_{t}^{2} \\
& +\left(A_{\overline{\phi_{y}}}+A_{\overline{\phi_{2 y}}}\right)^{3} c_{y}^{0} \delta_{t}^{3},
\end{aligned}
$$

such that the $\mathrm{n}^{\text {th }}$ iteration is given by

$$
\bar{\delta}_{c}^{n}=\sum_{i=1}^{n+1}\left(A_{\overline{\phi_{y}}}+A_{\overline{\phi_{2 y}}}\right)^{i} \delta_{t}^{i} c_{y}^{0} .
$$

The basic criterion for stability is that a small perturbation in the solution will decrease or remain constant in value through time, $t \rightarrow \infty$. Now, since in the above sum, the order of the terms (with respect to $\delta_{t}$ ) increases with $i$, we can form a preliminary estimate of the perturbation's growth using only the first $i=1$ terms, namely

$$
c_{x_{i}, y_{j}}^{1}=c_{x_{i}, y_{j}}^{0}+\left(-\phi_{y_{j}} c_{x_{i}, y_{j}}^{0}+2^{P+1} \phi_{2 y_{j}} c_{x_{i}, 2 y_{j}}^{0}\right) \cdot \delta_{t}
$$

and so we get the following condition for stability

$$
\phi_{y_{j}} c_{x_{i}, y_{j}}^{n} \geq 2^{P+1} \phi_{2 y_{j}} c_{x_{i}, 2 y_{j}}^{n} .
$$

Given that $0 \leq \phi \leq 1$, we have that for stability

$$
\begin{array}{ll}
\lim _{\delta_{y_{j} \rightarrow 0} \rightarrow 0} \lim _{y_{j} \rightarrow 0} c_{x_{i}, y_{j}}^{n}=\infty, & \forall n \in[0, N), \\
\lim _{\delta_{y_{j}} \rightarrow 0} \lim _{y_{j} \rightarrow \infty} c_{x_{i}, y_{j}}^{n}=0, & \forall n \in[0, N) .
\end{array}
$$

Further, concerning the structural dynamics, denoting now by $\overline{\bar{\delta}}_{c ; x_{i}, y_{j}}^{n}$ the change in the cancer cell distribution $c$ due to the $y$-flux of the system at a 
given spatio-structural position $\left(x_{i}, y_{j}\right)$ during a time interval $\left[n \delta_{t},(n+1) \delta_{t}\right]$, we have that

$\overline{\bar{\delta}}_{c ; x_{i}, y_{j}}^{0}:=A_{y} c_{y}^{0} \cdot \delta_{t}=\left(\frac{1}{2 \delta_{y}}\left(b_{y_{j-1}}-d_{y_{j-1}}\right) c_{x_{i}, y_{j-1}}^{0}-\frac{1}{2 \delta_{y}}\left(b_{y_{j+1}}-d_{y_{j+1}}\right) c_{x_{i}, y_{j+1}}^{0}\right) \cdot \delta_{t}$.

Therefore, if we have that $b, d$ are proportional to $y$, then we can extract the modified binding and unbinding rates $\breve{b}$ and $\breve{d}$ as

$$
b_{y_{j}}^{n}\left(y_{j}, \mathbf{m}^{n}\right)=\breve{b}\left(\mathbf{m}^{n}\right) \cdot y_{j} \text { and } d_{y_{j}}(y)=\breve{d} \cdot y_{j},
$$

and so we can then write

$$
\overline{\bar{\delta}}_{c ; x_{i}, y_{j}}^{0}=\left(y_{j-1} c_{x_{i}, y_{j-1}}^{0}-y_{j+1} c_{x_{i}, y_{j+1}}^{0}\right) \cdot \frac{1}{2 \delta_{y}}\left(\breve{b}^{0}-\breve{d}^{0}\right) \cdot \delta_{t},
$$

whose stability is ensured by having either

$$
\breve{b} \leq \breve{d},
$$

or

$$
c_{x_{i}, y_{j-1}}^{0} \leq \frac{y_{y_{j+1}}}{y_{y_{j-1}}} c_{x_{i}, y_{j+1}}^{0}, \quad \forall x_{i}, y_{j} .
$$

Thinking biologically about the ramifications of the former constraint, (53a), this would mean that the unbinding of the molecular species involved was more frequent than the binding of these species which would imply a relationship of affinity that approaches 0 . Although these exist biologically, the considered system is one in which the binding of molecular species plays a major role in the metabolic processes of the cell and one can thusly disregard (53a) from consideration as trivial. Therefore, we consider here only (53b) as viable.

From (48), (49), and (53b), however, we have a contradiction and therefore, the system must be unstable with neither an absolute nor a convective instability. The instability present is absolute in its source but convective in its requirement and behaviour.

For the stability of the equation in $v$ of the discretised 1D-spatio 1Dstructular system (3), from (43) we observe first that $0 \leq c_{x_{i}, y_{j}}^{n} \leq 1$ and $0 \leq m_{k ; x_{i}, y_{j}}^{n}, \forall k \in\{1, \ldots, q\}$. It is then trivial to show the following eigenvalues relations

$$
\begin{aligned}
& \lambda_{\operatorname{diag}\left(c_{x_{i}, y_{j}}^{n}\right)}=c_{x_{i}, y_{j}}^{n} \geq 0 \\
& \lambda_{\operatorname{diag}\left(m_{k ; x_{i}}^{n}\right)}=m_{k ; x_{i}}^{n} \geq 0, \forall k \in\{1, \ldots, q\} \\
& \lambda_{B_{x} \leq 0} \leq
\end{aligned}
$$

where $\lambda_{\operatorname{diag}\left(c_{x_{i}, y_{j}}^{n}\right)}$ denote the eigenvalues of $\operatorname{diag}\left(c_{x_{i}, y_{j}}^{n}\right), \lambda_{\operatorname{diag}\left(m_{k ; x_{i}}^{n}\right)}$ are the eigenvalues of $\operatorname{diag}\left(m_{k ; x_{i}}^{n}\right)$, and $\lambda_{B_{x}}$ represent the eigenvalues of $B_{x}$. Therefore, using a similar notation, since for the eigenvalues of $B_{\phi}$, we have that $\lambda_{B_{\phi}} \geq 0$, given smooth solutions for $c_{x}^{n}$ and $m_{x}^{n}$, we finally obtain that the solutions for $v_{x}^{n}$ will remain smooth and stable. 
Finally, using the similar eigenvalue notation, for the stability in the equations for $\mathbf{m}$, we obtain that the eigenvalues for $C^{n}(\tilde{g}), \delta_{m}$ and $\tilde{\psi}_{m}$ have the properties

$$
\begin{aligned}
& \lambda_{C^{n}(\tilde{g})} \geq 0, \\
& \lambda_{\delta_{\mathbf{m}}}=\gamma_{\mathbf{m}}, \\
& \lambda_{\tilde{\psi}_{\mathbf{m}}} \geq 0 .
\end{aligned}
$$

Moreover, we can also observe that eigenvalues of $D_{\phi}$ have the property that

$$
\lambda_{D_{\phi}} \leq 0 \quad \text { iff } \quad \lambda_{\tilde{\psi}_{\mathbf{m}}} \leq \gamma_{\mathbf{m}}+\varepsilon \lambda_{C^{n}(\tilde{g})}
$$

where for convenience we used the vector convention in writing the above inequality, which simply means that the inequality is respected per each component. Thus, we have that either $(1) \tilde{\psi}_{\mathbf{m}}$ is proportional to $\varepsilon C^{n}(\tilde{g})$ or $(2) \tilde{\psi}_{\mathbf{m}}$ is proportional to $C^{n}\left(I_{r}\right)$. For case (1), if we let $k_{\psi, 1}$ be the proportionality constant within the relation $\psi_{\mathbf{m}}$, then we can write that there must exist some values for $C_{x}^{n}(\tilde{g})$ at which

$$
\begin{aligned}
k_{\psi, 1} \varepsilon \lambda_{C^{n}(\tilde{g})} & \leq \gamma_{\mathbf{m}}+\varepsilon \lambda_{C^{n}(\tilde{g})} \\
\left(k_{\psi, 1}-1\right) \varepsilon \lambda_{C^{n}(\tilde{g})} & \leq \gamma_{\mathbf{m}} \\
\left(k_{\psi, 1}-1\right) \varepsilon C^{n}(\tilde{g}) & \leq \gamma_{\mathbf{m}}
\end{aligned}
$$

where we used the same vector convention as in (56). Thus, using integration by parts, we can write

$$
\left(k_{\psi, 1}-1\right) \varepsilon\left(\tilde{g} C^{n}(I)-(\breve{b}-\breve{d}) y_{r} C^{n}\left(I_{r}\right)\right) \leq \gamma_{\mathbf{m}} .
$$

For case (2), if we let $k_{\psi, 2}$ be the proportionality constant within the relation $\psi_{\mathbf{m}}$ then we can write that there must exist some values for $C^{n}\left(I_{r}\right)$ at which

$$
\begin{aligned}
k_{\psi, 2} \lambda_{C^{n}\left(I_{r}\right)} & \leq \gamma_{\mathbf{m}}+\varepsilon \lambda_{C^{n}(\tilde{g})} \\
k_{\psi, 2} C^{n}(I) & \leq \gamma_{\mathbf{m}}+\varepsilon\left(\tilde{g} C^{n}(I)-(\breve{b}-\breve{d}) y_{r} C^{n}\left(I_{r}\right)\right) \\
k_{\psi, 2} C^{n}\left(I_{r}\right) & \leq \gamma_{\mathbf{m}}+\varepsilon\left(\tilde{g}-(\breve{b}-\breve{d}) y_{r}\right) C^{n}\left(I_{r}\right)
\end{aligned}
$$

where $\tilde{g} \geq(\breve{b}-\breve{d}) y_{r}$. Therefore, given sufficiently large values for $C^{n}\left(I_{r}\right)$, given by

$$
C^{n}\left(I_{r}\right) \leq \frac{\gamma_{\mathbf{m}}}{k_{\psi, 2}-\varepsilon\left(\tilde{g}-(\breve{b}-\breve{d}) y_{r}\right)},
$$

the solutions for $m_{i}^{n}$ will be unstable with

$$
\lim _{n \rightarrow \infty} m_{i}^{n}=\infty \quad \forall i \text { where } \psi_{m_{i}}=\psi_{m_{i}}\left(c_{x}^{n}, \cdot\right) .
$$

For sufficiently low values of $C^{n}\left(I_{r}\right)$, given by the contrary argument to (60), the solutions for $m_{i}^{n}$ will be stable with $m_{i}^{n}=0$. 


\section{Numerical Results and Simulations in 1D-Spatial Case}

The parameters considered throughout this work are chosen to be consistent with those set out in Domschke et al. [41], and other previous models [51], and are detailed in Appendix A.

Numerical results generated by running the test case, and finite difference scheme for the system, through MATLAB are given below (Fig. 1-9). Several different cases are simulated in order to numerically verify the validity of proposed changes to the system and in order to perform analyses of the system using numerically generated graphic results:

For the 1D-spatial, 1D-structural case the associated model (3), was explored numerically in the presence of initial conditions for $c(t, x, y)$ for $t=0$, given by

$$
c_{0}(x, y):=c(0, x, y)=\exp \left[-100\left(x^{2}+4\left(y-\frac{1}{4}\right)^{2}\right)\right],
$$

and the homogeneous ECM conditions

$$
v_{0}(x):=v(0, x)=1-\int_{\mathcal{P}} c(0, x, y) d y .
$$

\section{1 uPA in the absence of PAI-1: 1D-Spatial 1D-Structural Results}

One characteristic of the numerical solution, which has not previously been observed, is that of the partial travelling wave translation in the structural dimension (Fig. 1). That is to say that the proliferative terms lead to the travelling wave being depleted and replaced, to a greater extent at a lower value for $y$. These features shall be henceforth referred to as structural " $y$-waves" and is an essential feature in understanding the dynamics of such systems, given their recurrence in all domains. There is not sufficient evidence in the biological literature to verify that this is the case or to contradict this result.

The hyper-affinity binding also results in the behaviour of "replicative $y$ trapping" (referring to the behaviour of $c$ collection at the upper $y$ boundary as $y$-trapping and the proliferative duplication at $\frac{1}{2} y$ as replicative of this $y$ trapping) behaviour producing a discontinuity that fails to allow the system to continue the migration of $c$ through $\mathcal{P}$ and raises significant questions of the biological efficacy of this system when coupled to assumptions of equal mitosis. Again, this results from the binding a production of these species occurring at far higher rates than the unbinding or degradation of these species.

The $y$-waves actually caused a resultant $x$-resolved profile, $C(t, x)$, which was itself not smooth (Fig. 1); this is a ramification of the proliferative contribution to the replication of steep gradient profiles. One must observe that, within the discrete space, the proliferative term necessarily means that any gradient is replicated with a proliferative constant, $\mu_{c}$.

Further, one observes an sharp spiking behaviour that occurs only at the boundary, which can be directly observed for $t=50$ (Fig. 1c). The source of 

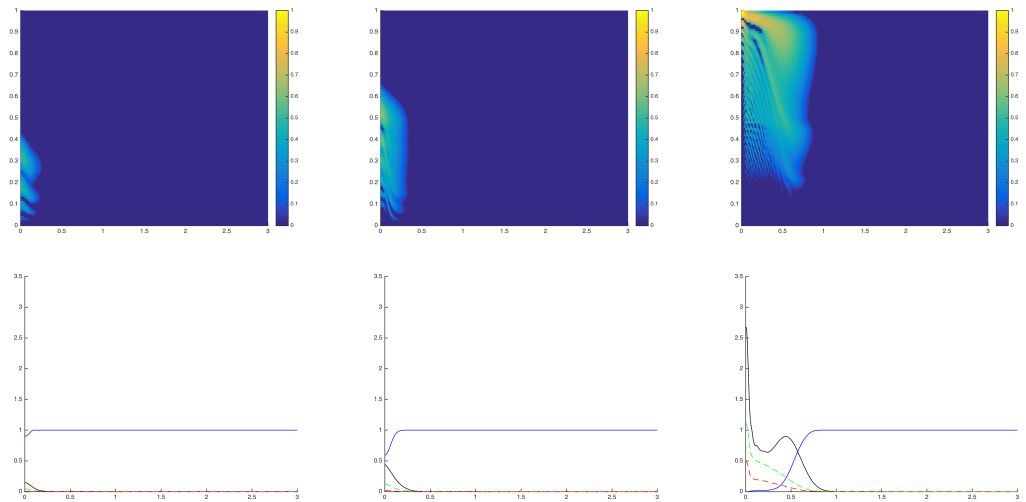

(b) $t=20$

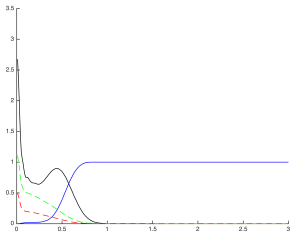

(a) $\mathrm{t}=10$

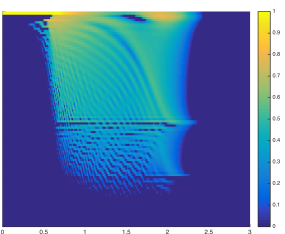

(c) $\mathrm{t}=40$
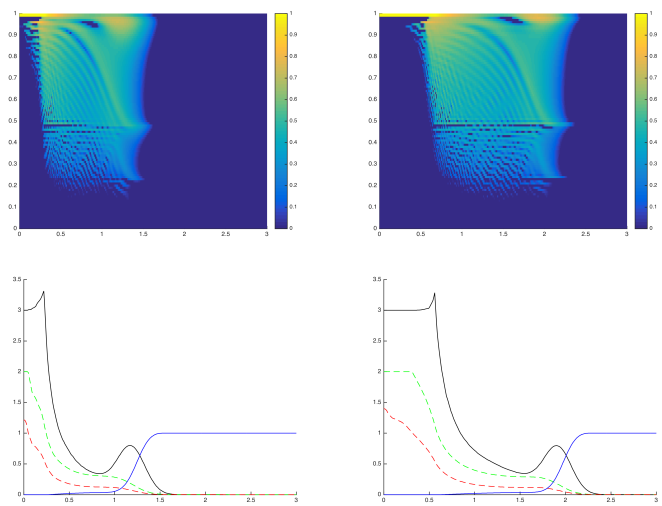

(e) $\mathrm{t}=80$
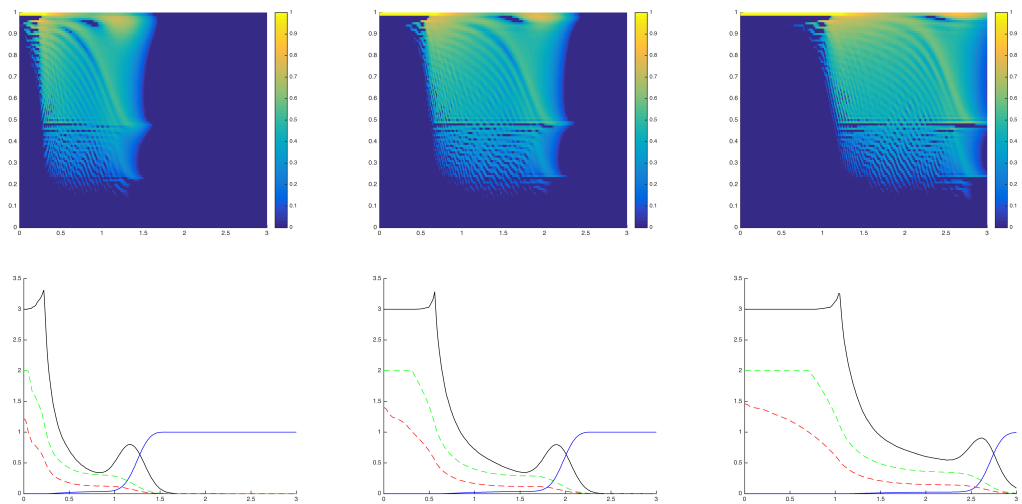

(d) $\mathrm{t}=60$
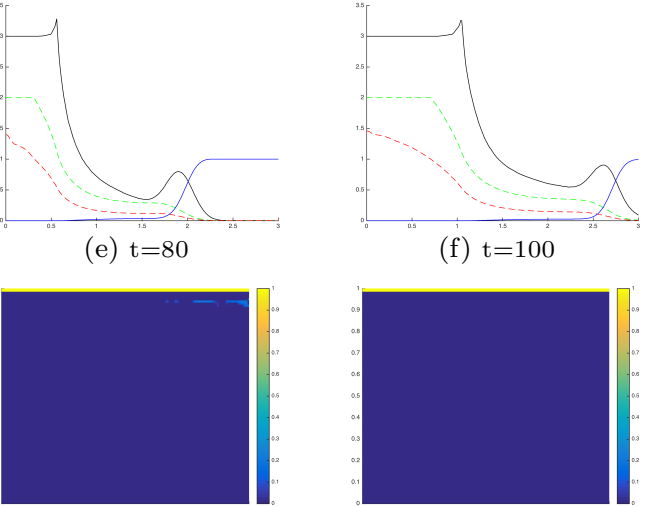

(f) $\mathrm{t}=100$
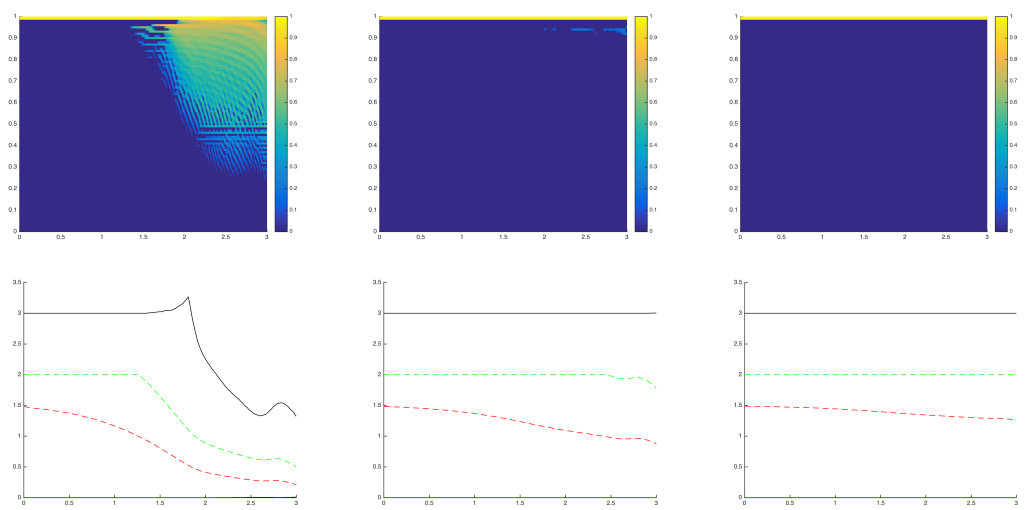

(g) $t=120$

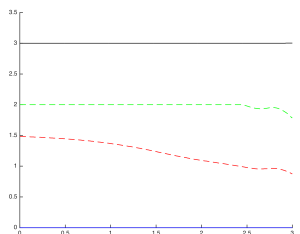

(h) $\mathrm{t}=140$

(i) $\mathrm{t}=160$

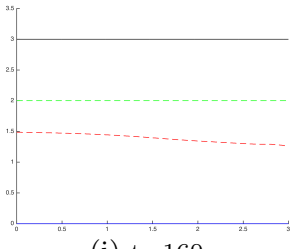

Fig. 1: Numerical 1D-spatial 1D-structural results generated from simulation of the system (3), with $c$ plotted in the x- and y-dimension (top), with values for $C$ (black), $v$ (blue), $m_{b, 1}$ (green dashed), and $m_{f, 1}$ (red dashed) plotted spatially (bottom).

this spiking is not clear, since it occurs to a lesser extent for other values of $(t, x)$, but could be due to the gradient-guided dissipation occurring only one 
side of the spike. In other words, the accuracy of the estimate of the double derivative is lessened by the fact that the peak occurs on the boundary and one can obtain information about the local features on only one side of the peak. It is also possible that this results from the contribution of the chemotaxis to the molecular species, on the bulk of the population, and the haptotaxis, on the exterior of the population.

There exists, however, some biological evidence to corroborate this behaviour as a natural process occurring due to the difference between forces between cell-cell junctions and cell-ECM based motility. For instance, Yamaguchi et al. [101] report the phenomenon of differential behaviours between 'leader' and 'follower' cells during collective cell migration, often resulting in a clustered cell subpopulation leading the migration of the tumour's boundary. Likewise, in vivo experimentation (necessarily invoking the heterogeneity of the underlying migratory substrate) has demonstrated breakaway clusters of cells which develop anterior to the invasive front [23].

\section{Numerical Results in 2D-Spatial Cases}

Proceeding in a similar manner to the $1 \mathrm{D}$-spatial case, also in the 2D-spatial cases, with the appropriate $1 \mathrm{D}$ - or $2 \mathrm{D}$-structural domain $\mathcal{P}$, we we assume equal spatial and structural step size $\delta_{x_{i}}:=\delta_{y_{j}}:=\delta_{x}, i, j \in\{1,2\}$ and an equal number $r \in \mathbb{N}^{*}$ of collocation points in both spatial and structural dimensions, and in the following we will proceed to discretise $\mathbf{c}, \mathbf{v}$, and $\mathbf{m}$ at any given time node $n \delta_{t}$, with $n \in \mathbb{N}$.

For the 2D-spatial 1D-structural model, numerical results have been obtained for the initial condition for $c(t, x, y)$ which are the extension of $(62)$, and in this case are given by

$$
c_{0}(x, y):=c(0, x, y)=\exp \left[-100\left(\|x\|_{2}^{2}+4\left(y-\frac{1}{4}\right)^{2}\right)\right],
$$

Furthermore, for the ECM, we use both the homogeneous initial conditions given in (63) and a new set of heterogeneous initial conditions given as in [5], namely:

$$
\begin{aligned}
\left(x_{1}, x_{2}\right) & :=\frac{1}{3}\left(x+\frac{3}{2}\right) \in[0,1]^{2} \text { for } x \in \mathcal{D}, \quad \zeta:=6 \pi, \\
h\left(x_{1}, x_{2}\right) & :=\frac{1}{2}+\frac{1}{2} \sin \left(\frac{\zeta x_{1}}{x_{2}+1}\right) \sin \left(\zeta x_{1} x_{2}\right) \sin \left(\frac{\zeta\left(1-x_{1}\right)}{x_{2}+1}\right) \sin \left(\zeta\left(x_{1}-1\right)\left(x_{2}-1\right)\right), \\
v_{0}(x) & :=v(0, x)=\min \left\{h\left(x_{1}, x_{2}\right), \frac{1-\mathfrak{v}_{c} C(0, x)}{\mathfrak{v}_{v}}\right\} .
\end{aligned}
$$

Finally, for the 2D-spatial 2D-structural model, numerical results have been obtained for the appropriate extension of the initial conditions for $c(t, x, y)$ considered in (62) and (64) which in this case recast as follows:

$$
c_{0}(x, y):=c(0, x, y)=\exp \left[-100\left(\|x\|^{2}+4\left\|\left(y-\left[\frac{1}{4}, \frac{1}{4}\right]^{T}\right)\right\|^{2}\right)\right],
$$

as well as the homogeneous and heterogeneous ECM initial conditions given in (63) and (65). 
5.1 uPA in the absence of PAI-1: 2D-Spatial 1D-Structural Results

Results from the simulations were consistent with the 1D-spatial 1D-structural case but varied widely due to the effect of the ECM on the cancer species.

Results for the 2D-spatial 1D-structural system for lower binding values are given (Fig. $2 \& 3$ ). For $c$, the the spatial distribution of the tumour in 2-spatial dimensions and isosurface figure in a 2D-spatial 1D-structural domain (in the absence of PAI-1) are displayed to attempt to give the fullest impression of the progress of the cancer through the spatial and structural domains in time. One can see, again, the $y$-wave behaviour in the 2D-spatial system with mushroomlike forms replicating themselves at progressively lower values for $y$. A typical symmetric cancer cluster grows into the spatial domain, giving higher values for concentration at the upper boundary of $\mathcal{P}$ towards the epicentre of the cancer cluster.

In biological terms, this indicates that the more established, inner, portion of the tumour will likely have a higher bound population of molecules that the boundary, outer, portion. This result is counter-intuitive since, given that these bound species are more effective at degrading the extracellular matrix, a theoretical postulation might lead one to believe that these species would exist to a greater extent on the boundary. This is either a flaw within the application of the model or may provide an interesting observation about the efficiency of natural biological cancers.

Biological evidence does exist, on the other hand, to support the claim that both uPAR, and consequently surface bound uPA, are more highly concentrated towards the interior of invasive cell structures. Invading $\mathrm{T}$ lyphocytes have been reported to exhibit such internally high expression levels, with only individuated exterior cells expressing high levels of uPARs [16]. A biological spatio-temporal model of tumour invasion reported high levels of uPA of the tumour's leading edge but also found extremely high levels on the invading mass' interior [2].

The $y$-waves occur within the 2D-spatial model also (Fig. 2-3), where one may observe graduated levels of binding for $c$. One can observe that the largest concentration of $c$ begins and remains at the epicentre of the population.

All results show a significant correlative relationship between the ECM levels, $v(t, x)$, and the destructive molecular species, $m_{f, 1}(t, x)$, and therefore a wave of ECM destruction follows closely behind the travelling wave of the cancer cell population. This is an indicator of the indirect relationship between the cancer growth mechanism and the cancer population itself (i.e. acting through the intermediate degradative protein species $\left.m_{f, 1}\right)$.

Results generated using a heterogeneous initial ECM density (Fig. 4-6) varied from the previously observed results with similar behaviour, nonetheless. One observes the spiking behaviours developing into a particularly defined wave front for the growing cancer population. Here, the boundary wave-like solution may be caused by the high affinity between the cancer cell population and the ECM. 

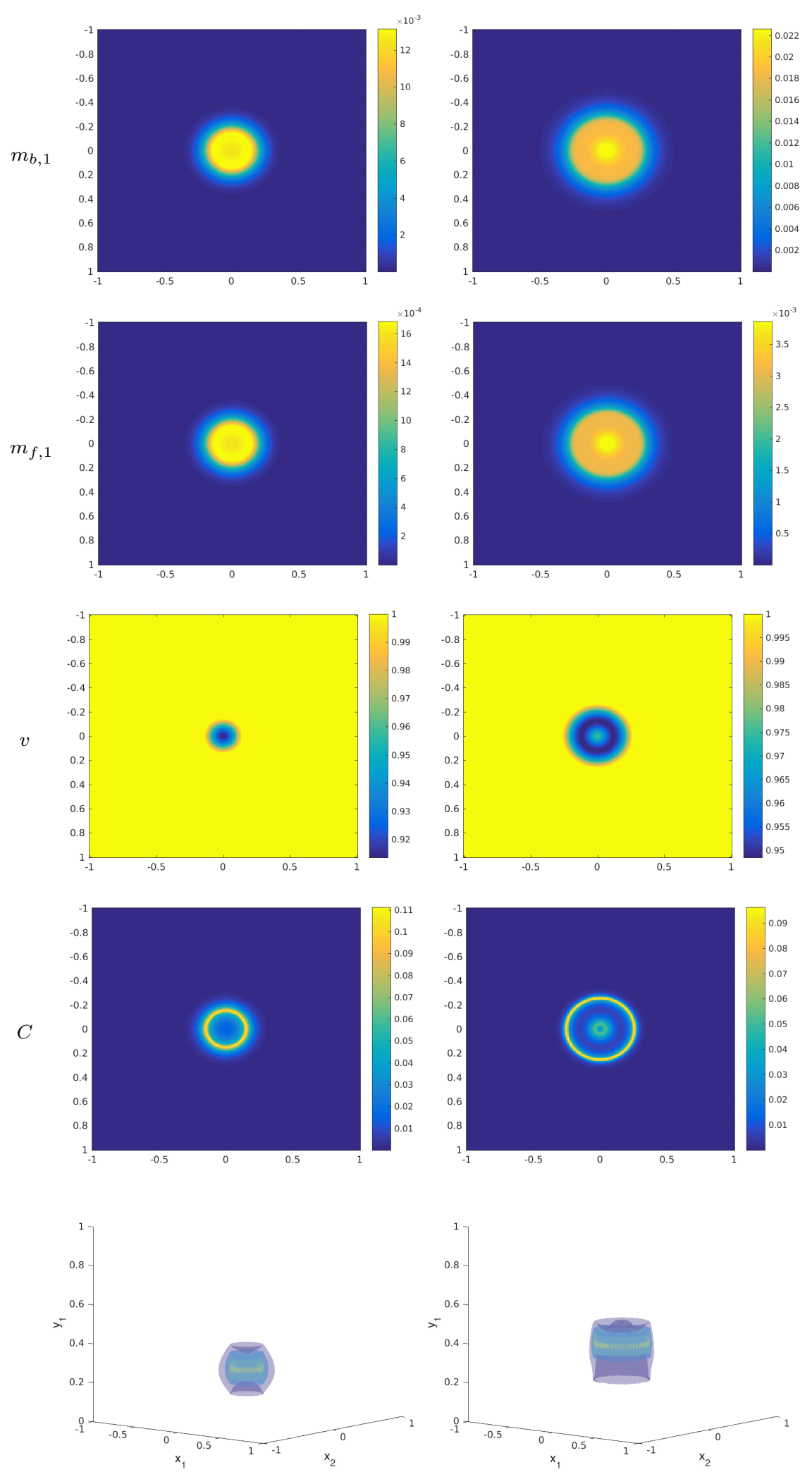

(a) $t=3$

(b) $\mathrm{t}=15$

Fig. 2: Numerical 2D-spatial 1D-structural results for (3) for homogeneous ECM, plotted at times $t \in\{3,15\}: m_{b, 1}($ row 1$), m_{f, 1}($ row 2) $v$ (row 3), $C$ (row 4), and $c$ (row 5) as an isosurface on the $2 \mathrm{D} x$-plane. 

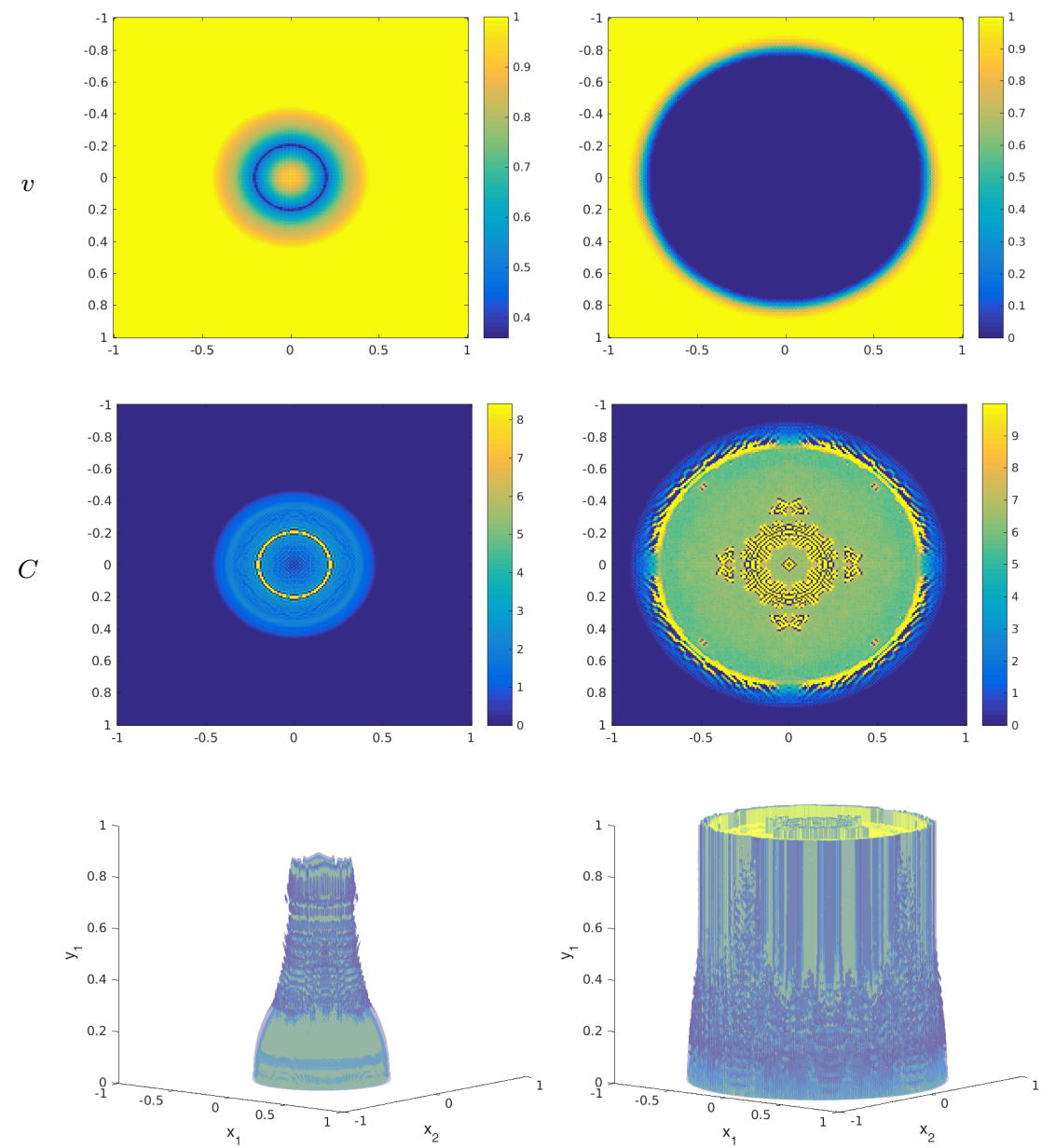

(a) $\mathrm{t}=35$

(b) $\mathrm{t}=50$

Fig. 3: Numerical 2D-spatial 1D-structural results for (3) for homogeneous ECM, plotted at times $t \in\{35,50\}: v($ row 1$), C($ row 2), and $c($ row 3$)$ as an isosurface on the $2 \mathrm{D}$ x-plane.

Although it is difficult to depict this behaviour, one again observes y-waves in the numerics for the behaviour at the interior of the cancer population. This is masked by the isosurface for the spiking shell of the cancer population. The $y$-waves play an important role in determining the initial behaviour of the cancer population, during growth and establishment of the perimeter. It is unclear wether these waves contribute to the dramatic change in behaviour and form $t \in(9,15)$.

Just as in the 1D- and 2D-spatial homogeneous-ECM cases, one can observe the initial spatial splitting of the cancer population. Unlike in the homogeneous case (Fig. 2-3), however, the heterogeneity appears to mediate the 

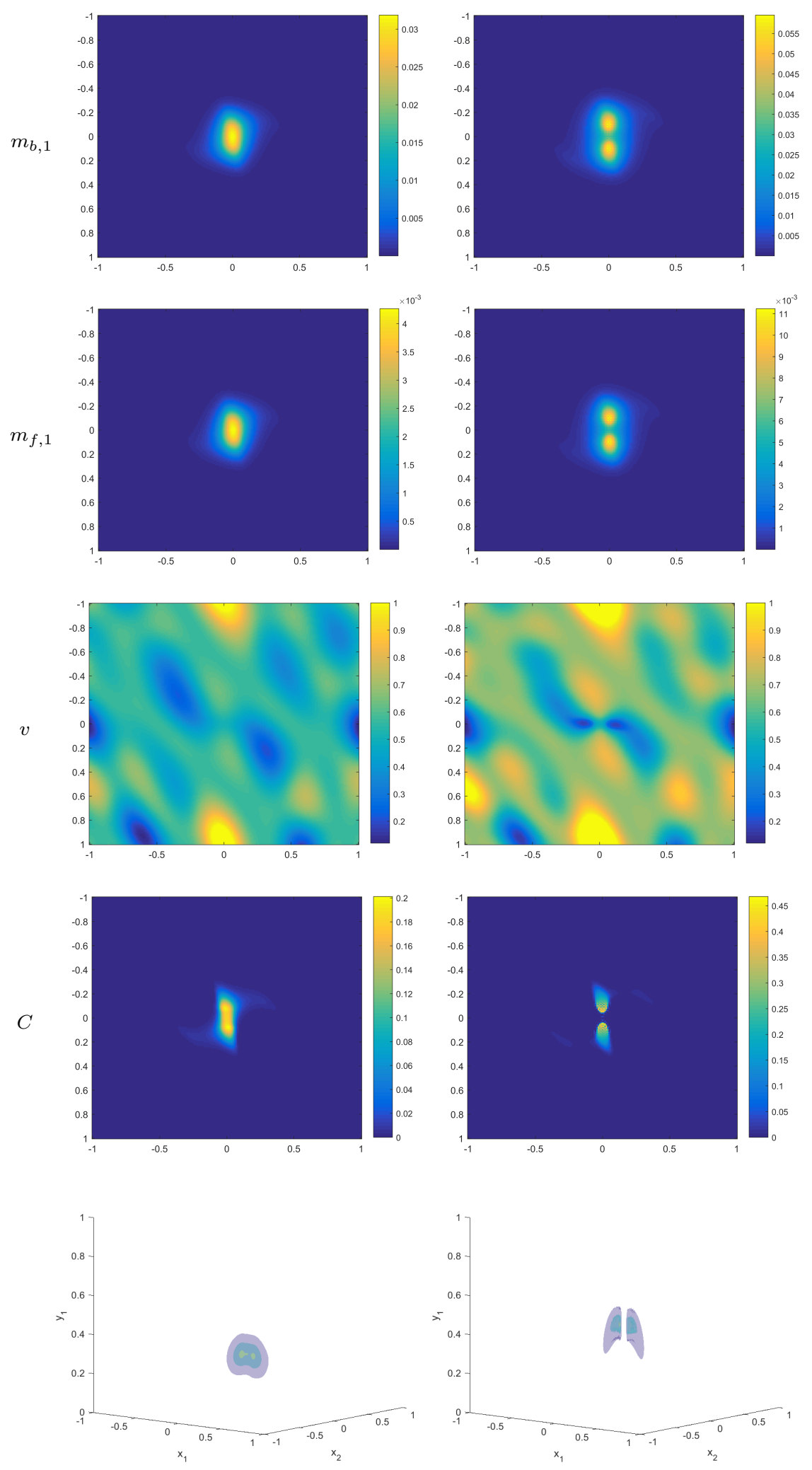

(a) $\mathrm{t}=3$

(b) $\mathrm{t}=9$

Fig. 4: Numerical 2D-spatial 1D-structural results for (3) for heterogeneous ECM, plotted at times $t \in\{3,9\}: m_{b, 1}\left(\right.$ row 1), $m_{f, 1}$ (row 2) $v$ (row 3), $C$ (row 4), and $c$ (row 5) as an isosurface on the $2 \mathrm{D}$ x-plane. 

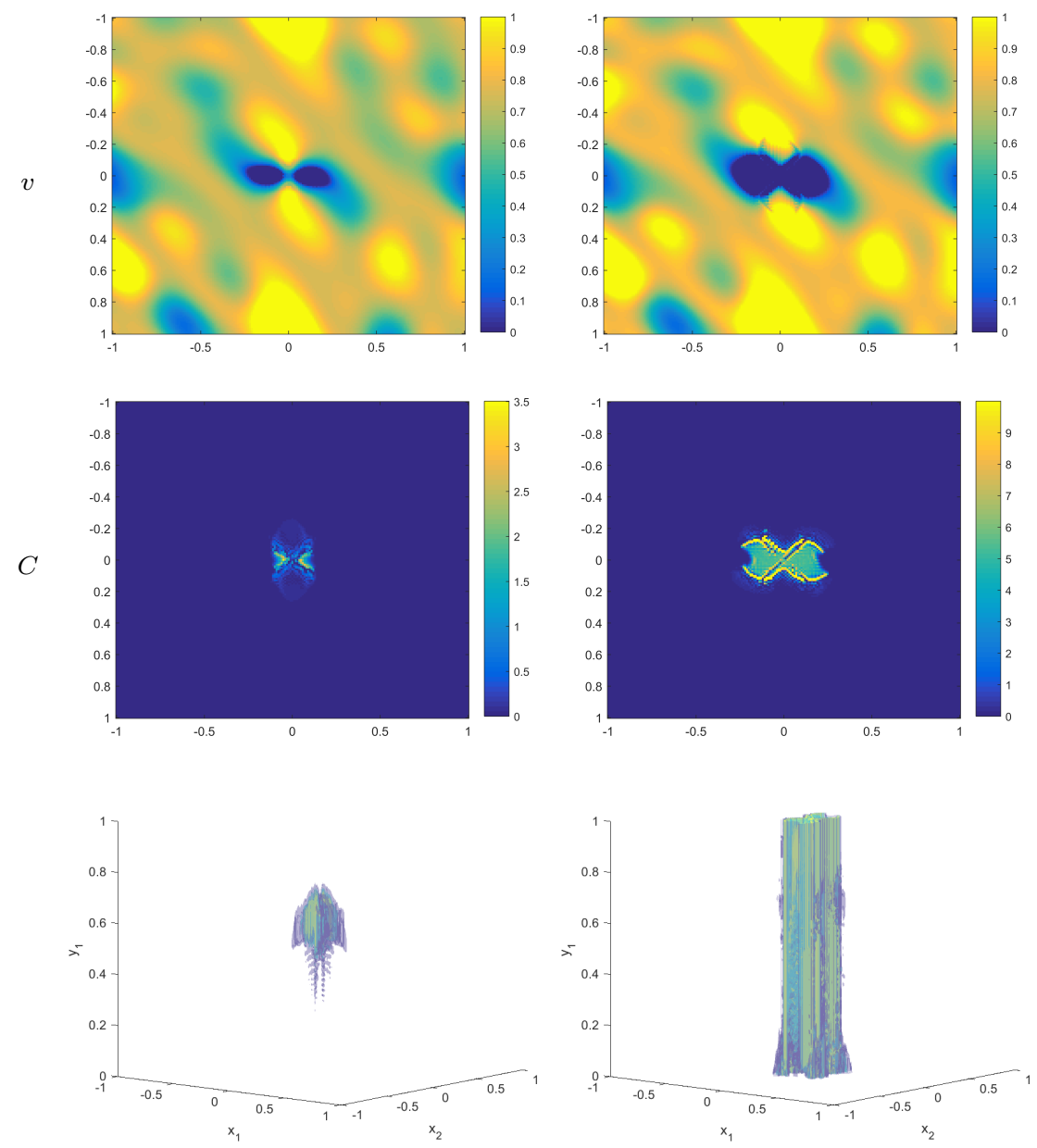

(a) $t=15$

(b) $t=20$

Fig. 5: Numerical 2D-spatial 1D-structural results for (3) for heterogeneous ECM, plotted at times $t \in\{15,20\}: v($ row 1$), C$ (row 2), and $c$ (row 3) as an isosurface on the 2D x-plane.

consolidation of the cancer subpopulations (Fig. 4b-5b) into the characteristic tumour that one associates with the biological paradigm.

Given that a periodic function is used to generate the ECM heterogeneity, it is not terribly surprising that the result produced $(t \leq 20)$ resembles that of a cyclic environment, with $180^{\circ}$ turn symmetry. Further results (not shown) were generated with an asymmetric, or with non radial symmetry with respect to the initial positioning of the tumour. These results again displayed an asymmetric splitting of the population (typically into two spatially distinct subpopulations) with the larger portion of the subpopulation migrating to those regions with the steepest ECM gradients. Given that all of these 

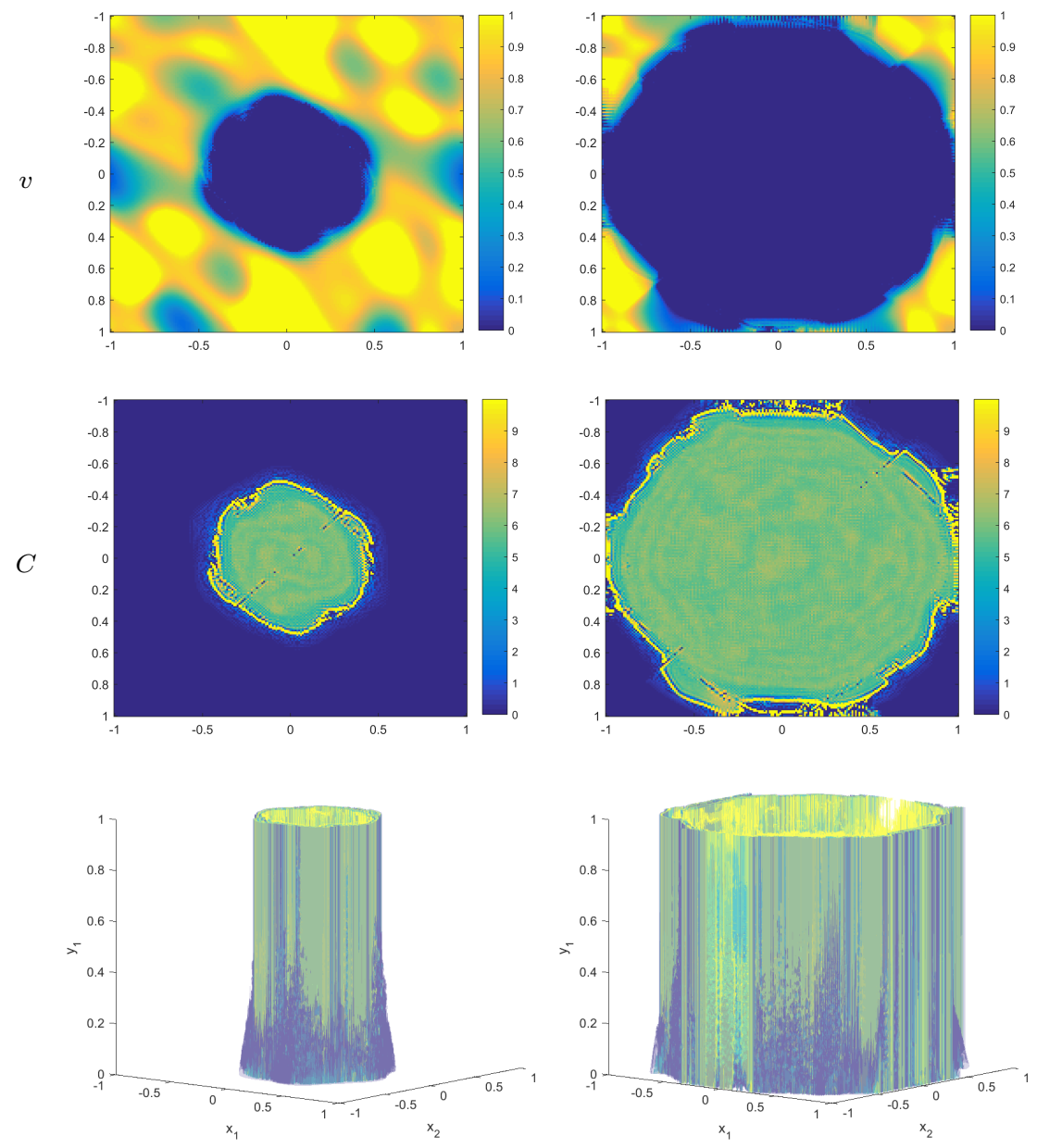

(a) $t=30$

(b) $\mathrm{t}=50$

Fig. 6: Numerical 2D-spatial 1D-structural results for (3) for heterogeneous ECM, plotted at times $t \in\{30,50\}: v($ row 1$), C$ (row 2), and $c($ row 3) as an isosurface on the 2D x-plane.

environments were normalised with respect to their overall nutritional capacity, the underlying ECM patterning has no significant bearing on the invasive success of the tumour.

Moreover, the cancer, in this 2D-spatial 1D-structural heterogeneous case, is particularly exploitative of its environment, protruding into areas of low ECM density before the diffusion of the molecular species through the more dense sections of the ECM allows the remainder of the population to follow. This feature of the cancer behaviour is repeated until total permeation of the spatial domain occurs. This also leads to the boundary of the cancer population becoming somewhat amorphous, as one observes with cancer in the natural, 
biological environment of the human tissue. It may be interesting to consider the case in which molecular species are more free to diffuse into areas of lower ECM density.

$5.2 \mathrm{uPA}$ in the presence of inhibitor PAI-1: 2D-Spatial 2D-Structural Results

Results generated using a heterogeneous initial ECM density were similar to those generated for the system with 1-structural dimension (in the absence of PAI-1, Fig. 7-9). One observes again that the spiking behaviours developing into a particularly defined wave front for the growing cancer population. Again, this is likely to be due to the attractive forces, leading to haptotaxis, between the cellular population and the ECM causing cells to chemotactically self-aggregate on the boundary of the tumour. This is also likely accentuated by the local inhibition of invasion (through PAI-1 binding), which contrasts the advancement of the tumour boundary and encourages hyper-localised behaviours.

All of those significant features, appearing in the case of 1D-structural dimension (in the absence of PAI-1), appear in those for 2-structural dimensions (in the presence of PAI-1), with the important difference being that the nature of the tumour in this 2D-structural case develops at a much slower rate. This is expected behaviour given that the introduction of a $2^{\text {nd }}$ structural dimension, in this case, corresponds to the introduction of an inhibitor to the degradative activation protein, uPA. The apparent rate of change in tumour growth can be approximately given by $\alpha_{m_{f, 1}}-\gamma_{m_{f, 1}}$ such that the rate by which the tumour is slowed down is equal to the binding ability of the inhibitor. This can be clearly seen in the differences in morphology at $t<20$ (Fig. 7-8).

\section{Discussion \& Conclusions}

In the case where we consider a homogeneous cancer population and ECM density (Fig. 2-3) one observes a logistic boundary that expands, unimpeded, to its maximal radius within the given domain. Within this one observes a lower peak that clearly continues to expand throughout the space with the $y$-wave behaviour (which can be observed within the raw data), although at much lower values for $C$. This is likely due to the initial conditions remaining constant with the addition of a spatial dimension, causing values for $c$ to be distributed and for $C$ to be reduced. Overall, these solution were in line with the 1D-spatial test-systems but not indicative of natural cancer behaviour.

Once we endow the cancer species with its natural habitat (the heterogeneous ECM) one observes behaviours absolutely characteristic of the biological system. These behaviours included the volume filling properties for the inside of the tumour; the aggressive behaviour of the cancer's perimeter; and the primary invasion into areas of lower tissue density. It is not clear whether or not the initial splitting of the cancer into two subpopulations is characteristic 

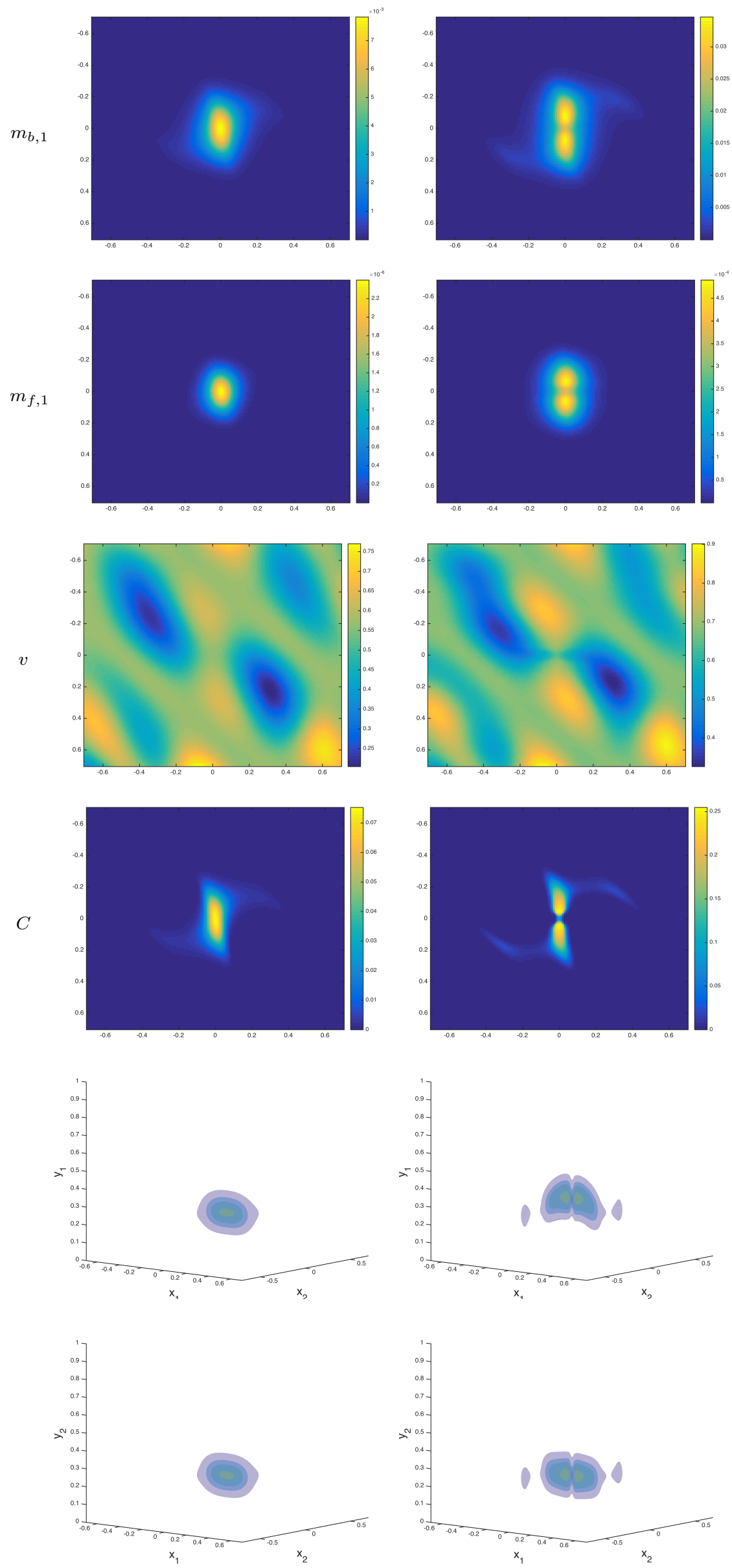

(a) $\mathrm{t}=3$

(b) $\mathrm{t}=9$

Fig. 7: Numerical 2D-spatial 2D-structural results for (3) for heterogeneous ECM, plotted at times $t \in\{3,9\}: m_{b, 1}($ row 1$), m_{f, 1}\left(\right.$ row 2), v (row 3),C (row 4), $\int_{[0,1]} c d y_{2}\left(\right.$ row 5) and $\int_{[0,1]} c d y_{1}$ (row 6 ) as isosurfaces on the $2 \mathrm{D} \mathrm{x}$-plane. 

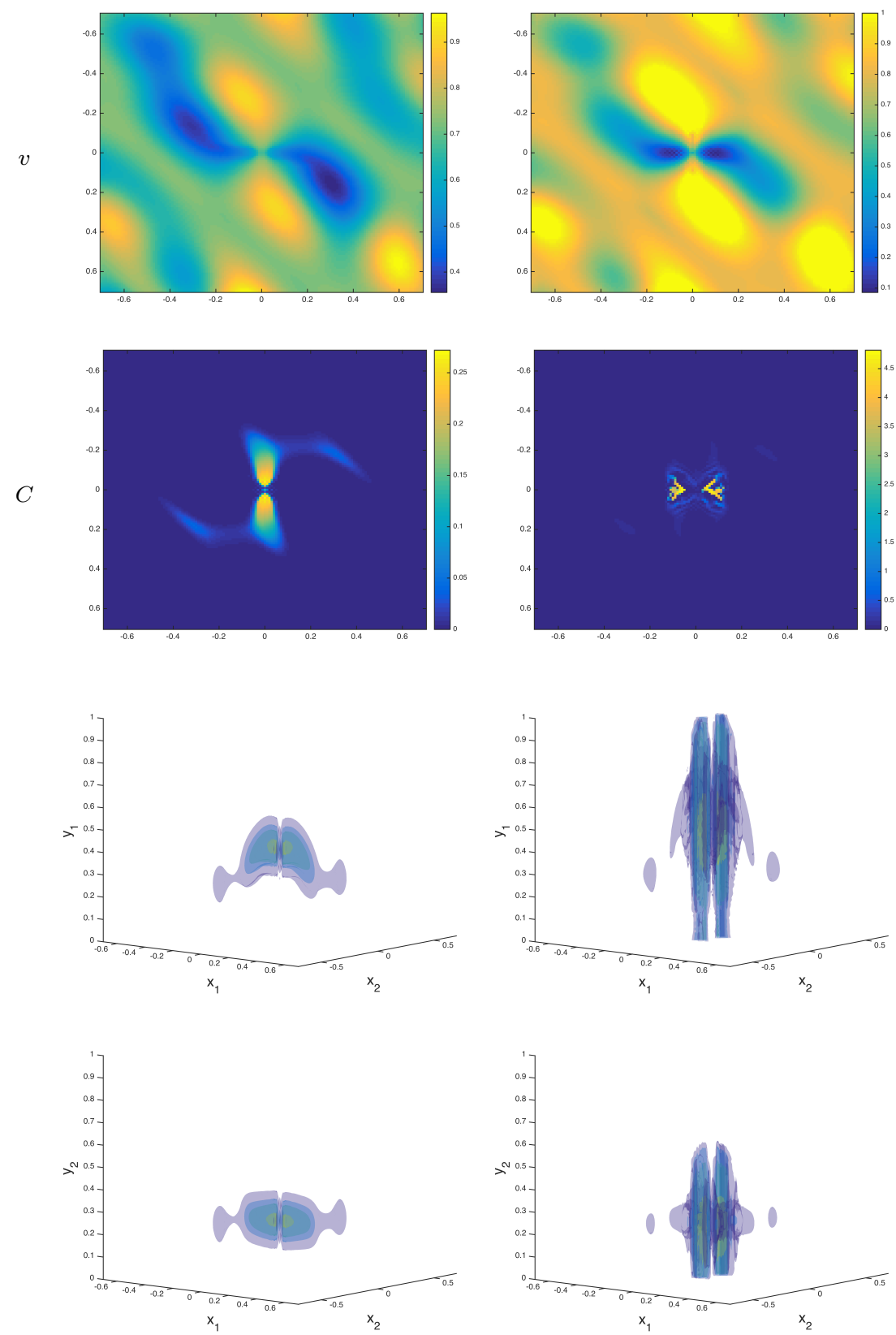

(a) $\mathrm{t}=12$

(b) $t=20$

Fig. 8: Numerical 2D-spatial 2D-structural results for (3) for heterogeneous ECM, plotted at times $t \in\{12,20\}: v($ row 1$), C$ (row 2), $\int_{[0,1]} c d y_{2}\left(\right.$ row 3) and $\int_{[0,1]} c d y_{1}$ (row 4) as isosurfaces on the $2 \mathrm{D} x$-plane. 

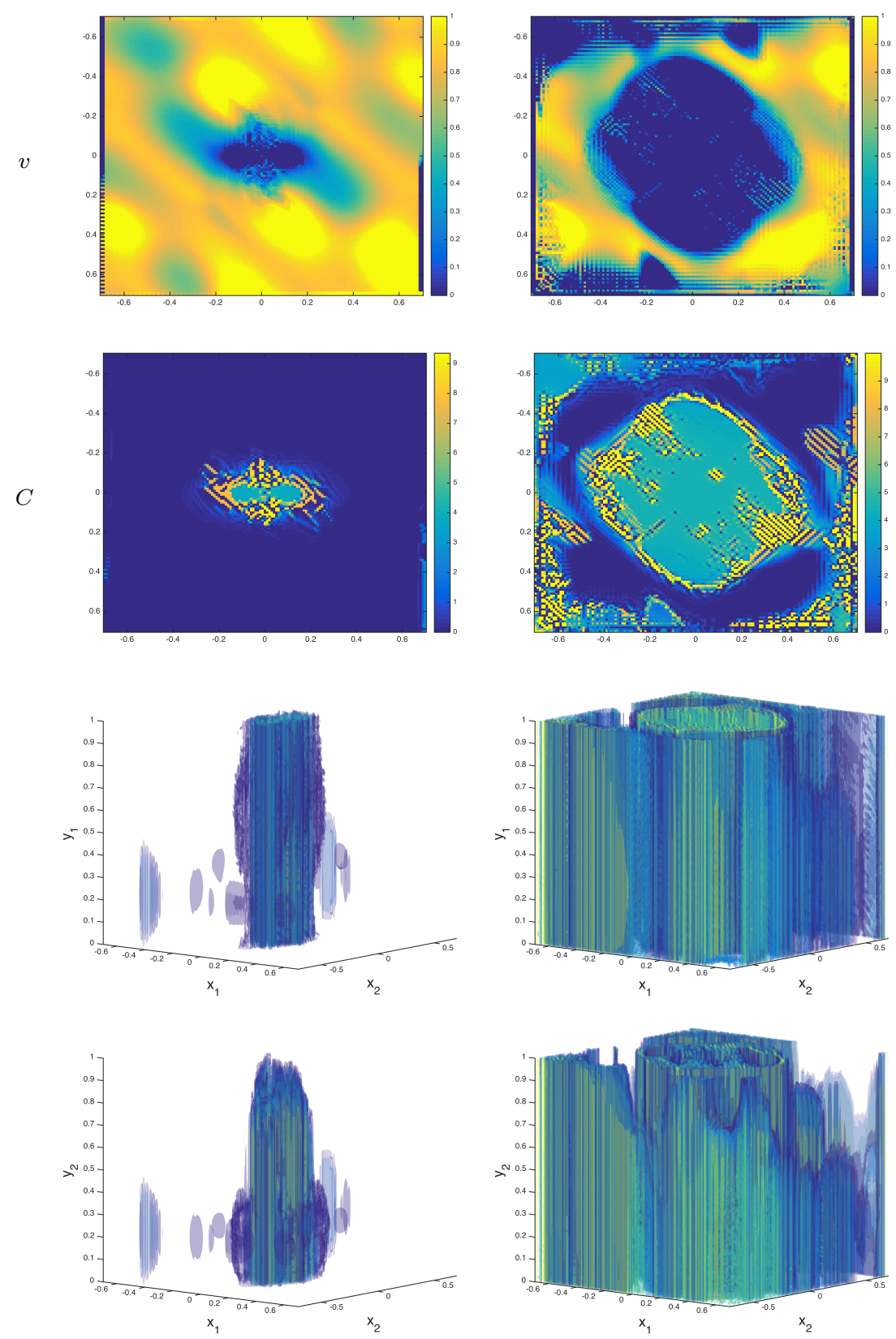

(a) $t=25$

(b) $t=35$

Fig. 9: Numerical 2D-spatial 2D-structural results for (3) for heterogeneous ECM, plotted at times $t \in\{25,35\}: v($ row 1$), C$ (row 2), $\int_{[0,1]} c d y_{2}\left(\right.$ row 3) and $\int_{[0,1]} c d y_{1}($ row 4) as isosurfaces on the $2 \mathrm{D} x$-plane. 
of nature but certainly is of the model, which is again likely a consequence of the initial conditions.

It can also be directly observed that the numerical solution is stable in its spatial dynamics but unstable in structural dynamics, which can be seen from the total permeation of $\mathcal{P}$ on the boundary of $c$ whilst the spatially considered cancer population, $C$, does not exhibit uncontrolled migration in $\mathcal{D}$. The areas of higher values for $C$ (Fig. 6) can be attributed to the fact that $y$-waves continue to exist within the body of the tumour and behave as in the 1D-spatial model.

The largest errors arise from the structural differentiation in the second term of the equation for $\frac{\partial c}{\partial t}$, where we incur second order errors as a result of the symmetric difference quotient approximation. This method is commonly used and there are no clear contenders to be used in its place since reaching a higher order approximation is far more difficult than incurring computational and processing penalties as a result of increasing the number of required arithmetic calculations.

We then have a third order error that results from the trapezoidal approximation on the local integral. This could, potentially, be improved by, for example, taking a higher order approximation, such as Simpson's rule polynomial approximation, but one necessarily has a trade off between accuracy and computational intensity.

On the whole, these errors tend to be small so long as $\delta_{x}$ and $\delta_{y}$ are kept sufficiently small, $\delta_{x}, \delta_{y} \leq 10^{-2}$.

The result of stability analysis was to confirm the conclusions of the numerical simulations that, notwithstanding ones ability to compensate for the errors produced, the system is unstable in $y$. Any perturbation induced with non-zero $y$-component will result in the exponential growth of the perturbation through the structure space.

This simply implies that for fine perturbations in $x$, one must have sufficiently low migration in $y$ so as to allow the profile to remain stable. This is particularly interesting when taken in combination with the numerical solutions which revealed explosive instabilities upon rapid migration to the $y$ boundary, or the boundary of $\overline{\mathcal{P}}, \partial \overline{\mathcal{P}}$.

Given that, for stability, one considers the magnitude (or absolute values) of the parameters involved, one has that $\mathbf{m}$ is unstable under all conditions and for small perturbation around any hypothetical stable solution. We also find that $v$ is stable at the cancer-ECM equilibrium such that, since we are interested in the cancer growth and destruction of its local environment, the system is stable, notwithstanding the instability in $\mathbf{m}$.

Numerical results show interesting behaviours, particularly with the introduction of $y$-waves, resulting from the structural considerations in $c$. One sees the expected characteristics of the aggressive cancer species considered, with smooth degradation of the ECM and concurrent advancement of the cancer 
species to encroach on the healthy tissue. One shows how various considerations as to how one could amend the system result in more reasonable and expected behaviours in the cancer species but how, ultimately, the results that most closely correlate with the biological paradigm require a rethink of the underlying assumptions for the system, as a whole.

The numerical results for the system may elucidate an interesting propensity of the biological cancer system to utilise unconventional mechanisms of invasion, under the influence of chemical inhibitors. Inhibiting the uPA system appears, in some way, to impede the uniform invasion of the stroma by this aggressive cancer species, whilst allowing the ECM to remain intact by reducing the cancer's ability to degrade collagen has allowed the cancer to more readily utilise haptotactic behaviours. Therefore, what one observes in the inhibitor system, in comparison to its counterpart, is a more sporadic distribution of cancer cells who invade but do not degrade the ECM (growing in areas where competition for space is reduced.

This gives rise to important biological decisions about how one treats and prevents the spread of these cells, since inhibition may cause greater clinical issues. The inhibitors fail to inhibit the initial devastation of the ECM by uPA but leave the ECM open to exploitation. This may be a survival mechanism utilised by cancer and may have been evolutionarily beneficial to it propensity to arise in the human body, rather than acting to the detriment of its progression.

\section{A Parameter Set}

$$
\begin{array}{cccccc}
c & : & D_{c}=10^{-4} & \xi_{v}=5 \times 10^{-2} & \xi_{1}=1 \times 10^{-3} & \mu_{c}=0.1 \\
& & S_{c c}=10^{-4} & S_{c v}=10^{-4} & \mathfrak{v}_{c}=0.524 & \mathfrak{v}_{v}=0.476 \\
\text { i-state } & : & & \varepsilon=0.1 & \beta=0.5 & \gamma_{y}=0 \\
v & : & & \gamma_{v}=10 & \mu_{v}=0.05 & \\
m_{b, 1} & : & D_{m_{b, 1}}=10^{-3} & \alpha_{m_{b, 1}}=0.1 & \gamma_{m_{b, 1}}=0.1 & \\
m_{b, 1} & : & D_{m_{b, 1}}=10^{-3} & \alpha_{m_{b, 1}}=0.1 & \gamma_{m_{b, 2}}=0.1 & \\
m_{f, 1} & : & D_{m_{f, 1}}=10^{-3} & \alpha_{m_{f, 1}}=0.5 & \gamma_{m_{f, 1}}=0.1 &
\end{array}
$$

\section{References}

1. Adam, J., Bellomo, N.: A Survey of Models for Tumor-Immune System Dynamics. Birkhäuser, Boston (1996)

2. Adam, L., Mazumdar, A., Sharma, T., Jones, T.R., Kumar, R.: A three-dimensional and temporo-spatial model to study invasiveness of cancer cells by heregulin and prostaglandin $\mathrm{e}_{2}$. Cancer Research 61, 81-87 (2001)

3. Al-Omari, J., Gourley, S.: Monotone travelling fronts in an age-structured reactiondiffusion model of a single species. Journal of Mathematical Biology 45(4), 294-312 (2002). DOI 10.1007/s002850200159 
4. Allen, E.J.: Derivation of stochastic partial differential equations for size- and agestructured populations. Journal of Biological Dynamics 3(1), 73-86 (2009). DOI 10.1080/17513750802162754

5. Andasari, V., Gerisch, A., Lolas, G., South, A.P., Chaplain, M.A.J.: Mathematical modeling of cancer cell invasion of tissue: biological insight from mathematical analysis and computational simulation. Journal of Mathematical Biology 63(1), 141-171 (2011). DOI 10.1007/s00285-010-0369-1

6. Anderson, A., Chaplain, M.A.J.: Continuous and Discrete Mathematical Models of Tumor-induced Angiogenesis. Bulletin of Mathematical Biology 60(5), 857-899 (1998)

7. Anderson, A.R.A., Chaplain, M.A.J., Newman, E.L., et al.: Mathematical Modelling of Tumour Invasion and Metastasis. Journal of Theoretical Medicine 2(2), 129-154 (2000)

8. Ayati, B.P.: A structured-population model of proteus mirabilis swarm-colony development. Journal of Mathematical Biology 52(1), 93-114 (2006). DOI 10.1007/ s00285-005-0345-3

9. Barinka, C., Parry, G., Callahan, J., et al.: Structural Basis of Interaction between Urokinase-type Plasminogen Activator and its Receptor. Journal of Molecular Biology 363(2), 482-495 (2006)

10. Basse, B., Ubezio, P.: A Generalised Age- and Phase-Structured Model of Human Tumour Cell Populations Both Unperturbed and Exposed to a Range of Cancer Therapies. Bulletin of Mathematical Biology 69(5), 1673-1690 (2007)

11. Bellomo, N., Li, N.K., Maini, P.K.: On the foundations of cancer modelling: Selected topics, speculations, and perspectives. Mathematical Models and Methods in Applied Sciences 18(4), 593-646 (2008)

12. Bellomo, N., Preziosi, L.: Modelling and mathematical problems related to tumor evolution and its interaction with the immune system. Mathematical and Computer Modelling 32, 413-452 (2000)

13. Benzekry, S., Lamont, C., Beheshti, A., et al.: Classical Mathematical Models for Description and Prediction of Experimental Tumor Growth. PLoS Computational Biology 10(8), e1003,800 (2014)

14. Bertuzzi, A., D'Onofrio, A., Fasano, A., Gandolfi, A.: Modelling cell populations with spatial structure: Steady state and treatment-induced evolution of tumour cords. Discrete and Continuous Dynamical Systems - Series B 4(1), 161-186 (2004)

15. Bhuvarahamurthy, V., Schroeder, J., Kristiansen, G., et al.: Differential gene expression of urokinase-type plasminogen activator and its receptor in human renal cell carcinoma. Oncology Reports 14(3), 777-782 (2005)

16. Bianchi, E., Ferrero, E., Fazioli, F., Mangili, F., Wang, J., Bender, J.R., Blasi, F., Pardi, R.: Integrin-dependent induction of functional urokinase receptors in primary t lymphocytes. Journal of Clinical Investigation 98(5), 1133-1141 (1996)

17. Binder, B.R., Mihaly, J., Prager, G.W.: uPAR - uPA - uPAI-1 interactions and signalling: A vascular biologist's view. International Journal for Vascular Biology and Medicine 97, 336 - 342 (2007)

18. Busenberg, S., Iannelli, M.: A class of nonlinear diffusion problems in age-dependent population dynamics. Nonlinear Analysis: Theory, Methods \& Applications 7(5), 501 - 529 (1983). DOI 10.1016/0362-546X(83)90041-X

19. Calsina, À., Saldaña, J.: A model of physiologically structured population dynamics with a nonlinear individual growth rate. Journal of Mathematical Biology 33(4), 335364 (1995). DOI 10.1007/BF00176377

20. Chaplain, M.A.J., Ganesh, M., Graham, I.G.: Spatio-temporal pattern formation on spherical surfaces: numerical simulation and application to solid tumour growth. Journal of Mathematical Biology 42(5), 387-423 (2001)

21. Chaplain, M.A.J., Lolas, G.: Mathematical modelling of cancer cell invasion of tissue: the role of the urokinase plasminogen activation system. Mathematical Models and Methods in Applied Sciences 11(2005), 1685-1734 (2005)

22. Chaplain, M.A.J., Lolas, G.: Mathematical modelling of cancer cell invasion of tissue: The role of the urokinase plasminogen activation system. Mathematical Models and Methods in Applied Sciences (2011) 
23. Chapman, A., del Ama, L.F., Ferguson, J., Kamarashev, J., Wellbrock, C., Huristone, A.: Heterogeneous tumour subpopulations cooperate to drive invasion. Cell Reports 8, 688-695 (2014)

24. Chapman, S.J., Plank, M.J., James, A., Basse, B.: A nonlinear model of age and sizestructured populations with applications to cell cycles. The ANZIAM Journal 49(02), 151 (2007)

25. Chaurasia, P., Aguirre-Ghiso, J.A., Liang, O.D., et al.: A Region in Urokinase Plasminogen Receptor Domain III Controlling a Functional Association with 5beta1 Integrin and Tumor Growth. Journal of Biological Chemistry 281(21), 14,852-14,863 (2006)

26. Clayton, D., Schifflers, E.: Models for temporal variation in cancer rates. I: Age-period and age-cohort models. Statistics in Medicine 6(4), 449-467 (1987)

27. Cushing, J.M.: An Introduction to Structured Population Dynamics, CBMS-NSF Regional Conference Series in Applied Mathematics, vol. 71. SIAM (1998). DOI 10.1137/1.9781611970005.ch2

28. Cusulin, C., Iannelli, M., Marinoschi, G.: Age-structured diffusion in a multi-layer environment. Nonlinear Analysis: Real World Applications 6(1), 207 - 223 (2005). DOI 10.1016/j.nonrwa.2004.08.006

29. Dan $\varnothing$, K., Andreasen, P., Grøndahl-Hansen, J., et al.: Plasminogen Activators, Tissue Degradation, and Cancer. Advances in Cancer Research 44, 139-266 (1985)

30. Dan $\varnothing$, K., Rømer, J., NIELSEN BOYE S., B.S., et al.: Cancer invasion and tissue remodeling-cooperation of protease systems and cell types. APMIS 107(1-6), 120-127 (1999)

31. Delgado, M., Molina-Becerra, M., Suárez, A.: A nonlinear age-dependent model with spatial diffusion. Journal of Mathematical Analysis and Applications 313(1), 366 380 (2006). DOI 10.1016/j.jmaa.2005.09.042

32. Delitala, M., Lorenzi, T.: Asymptotic dynamics in continuous structured populations with mutations, competition and mutualism. Journal of Mathematical Analysis and Applications 389, 439-451 (2012). DOI 10.1016/j.jmaa.2011.11.076

33. Delitala, M., Lorenzi, T., Melensi, M.: Competition between cancer cells and t cells under im- munotherapy: a structured population approach. ITM Web of Conferences 5, 5p (2015). DOI 10.1051/itmconf/20150500005

34. Deng, Q., Hallam, T.G.: An age structured population model in a spatially heterogeneous environment: Existence and uniqueness theory. Nonlinear Analysis: Theory, Methods \& Applications 65(2), 379 - 394 (2006). DOI 10.1016/j.na.2005.06.019

35. Di Blasio, G.: Non-linear age-dependent population diffusion. Journal of Mathematical Biology 8(3), 265-284 (1979). DOI 10.1007/BF00276312

36. Diekmann, O., Gyllenberg, M., Metz, J.A.J., Thieme, H.: The 'Cumulative' Formulation of (Physiologically) Structured Population Models. CWI (1992)

37. Diekmann, O., Heijmans, H.J.A.M., Thieme, H.R.: On the stability of the cell size distribution. Journal Of Mathematical Biology 19(2), 227-248 (1984)

38. Diekmann, O., Metz, J.A.J.: On the Reciprocal Relationship Between Life Histories and Population Dynamics, Lecture Notes in Biomathematics, vol. 100, chap. Frontiers in Mathematical Biology, pp. 263-279. Springer Berlin Heidelberg (1994)

39. Diekmann, O., Temme, N.M. (eds.): Nonlinear Diffusion Problems. No. 28 in MC Syllabus. Mathematisch Centrum, Amsterdam (1982)

40. Domschke, P., Trucu, D., Gerisch, A., et al.: Mathematical modelling of cancer invasion: Implications of cell adhesion variability for tumour infiltrative growth patterns. Journal of Theoretical Biology 361, 41-60 (2014)

41. Domschke, P., Trucu, D., Gerisch, A., et al.: Structured models of cell migration incorporating molecular binding processes. Journal of Mathematical Biology 75, 1517-1561 (2017). DOI 10.1007/s00285-017-1120-y

42. Dufau, I., Frongia, C., Sicard, F., Dedieu, L., et al.: Multicellular tumor spheroid model to evaluate spatio-temporal dynamics effect of chemotherapeutics: application to the gemcitabine/CHK1 inhibitor combination in pancreatic cancer. BMC Cancer 12(1), $15(2012)$

43. Duffy, M.J., Maguire, T.M., McDermott, E.W., et al.: Urokinase plasminogen activator: A prognostic marker in multiple types of cancer. Journal of Surgical Oncology 71(2), 130-135 (1999) 
44. Dyson, J., Webb, G.: A nonlinear age and maturity structured model of population dynamics i. basic theory. Journal of Mathematical Analysis and Applications 242, 93-104 (2000)

45. Dyson, J., Webb, G.: A nonlinear age and maturity structured model of population dynamics ii. chaos. Journal of Mathematical Analysis and Applications 242, 255-270 (2000)

46. Ellis, V., Dan $\varnothing$, K.: Potentiation of plasminogen activation by an anti-urokinase monoclonal antibody due to ternary complex formation. A mechanistic model for receptormediated plasminogen activation. The Journal of biological chemistry 268(7), 4806-13 (1993)

47. Fitzgibbon, W., Parrott, M., Webb, G.: Diffusion epidemic models with incubation and crisscross dynamics. Mathematical Biosciences 128(1-2), $131-155$ (1995). DOI 10.1016/0025-5564(94)00070-G

48. Galle, J., Hoffmann, M., Aust, G.: From single cells to tissue architecture - a bottomup approach to modelling the spatio-temporal organisation of complex multi-cellular systems. Journal of Mathematical Biology 58(1-2), 261-283 (2009)

49. Garroni, M.G., Langlais, M.: Age-dependent population diffusion with external constraint. Journal of Mathematical Biology 14(1), 77-94 (1982). DOI 10.1007/ BF02154754

50. Gatenby, R.A., Gawlinski, E.T.: A Reaction-Diffusion Model of Cancer Invasion. Cancer Res. 56(24), 5745-5753 (1996)

51. Gerisch, A., Chaplain, M.: Mathematical modelling of cancer cell invasion of tissue Local and non-local models and the effect of adhesion. Journal of Theoretical Biology 250(4), 684-704 (2008)

52. Godár, S., Hořejší, V., Weidle, U.H., et al.: M6P/IGFII-receptor complexes urokinase receptor and plasminogen for activation of transforming growth factor- $\beta 1$. European Journal of Immunology 29(3), 1004-1013 (1999)

53. Gurtin, M., MacCamy, R.: Diffusion models for age-structured populations. Mathematical Biosciences 54(1-2), 49 - 59 (1981). DOI 10.1016/0025-5564(81)90075-4

54. Gyilenberg, M., Webb, G.F.: A nonlinear structured population model of tumor growth with quiescence. Journal of Mathematical Biology 28, 671-694 (1990)

55. Gyllenberg, M.: Nonlinear age-dependent population dynamics in continuously propagated bacterial cultures. Mathematical Biosciences 62(1), $45-74$ (1982). DOI http://dx.doi.org/10.1016/0025-5564(82)90062-1

56. Gyllenberg, M.: The size and scar distributions of the yeast saccharomyces cerevisiae. Journal of Mathematical Biology 24(1), 81-101 (1986). DOI 10.1007/BF00275722

57. Gyllenberg, M., Webb, G.: Age-size structure in populations with quiescence. Mathematical Biosciences 86(1), 67 - 95 (1987). DOI 10.1016/0025-5564(87)90064-2

58. Hanahan, D., Weinberg, R.A.: The hallmarks of cancer: The next generation. Cell 144, 646-674 (2011)

59. Hsieh, Y.H.: Altruistic population model with sex ifference. In: O. Arnino, D.E. Axelrod, M. Kimmel (eds.) Mathematical Population Dynamics, Lecture Notes in Pure and Applied Mathematics, vol. 131. Marcel Dekker, Inc. (1991)

60. Huai, Q., Mazar, A.P., Kuo, A., Parry, G.C., et al.: Structure of human urokinase plasminogen activator in complex with its receptor. Science (New York, N.Y.) 311(5761), 656-9 (2006)

61. Huang, C.: An age-dependent population model with nonlinear diffusion in $\mathbf{R}^{n}$. Quart. Appl. Math. 52, 377-398 (1994)

62. Huyer, W.: A size-structured population-model with dispersion. Journal of Mathematical Analysis and Applications 181(3), 716 - 754 (1994). DOI 10.1006/jmaa.1994.1054

63. Khanna, M., Wang, F., Jo, I., et al.: Targeting multiple conformations leads to smal molecule inhibitors of the upar-upa protein-protein interaction that block cancer cell invasion. ACS Chemical Biology (2011)

64. Kimmel, M., Darzynkiewicz, Z., Arino, O., Traganos, F.: Analysis of a cell cycle model based on unequal division of metabolic constituents to daughter cells during cytokinesis. Journal of Theoretical Biology 637-664 (1984)

65. Kondraganti, S., Gondi, C.S., McCutcheon, I., et al.: RNAi-mediated downregulation of urokinase plasminogen activator and its receptor in human meningioma cells inhibits tumor invasion and growth. International Journal of Oncology 28(6), 1353-1360 (2006) 
66. Kunisch, K., Schappacher, W., Webb, G.: Nonlinear age-dependent population dynamics with random diffusion. Computers \& Mathematics with Applications 11(1-3), 155-173 (1985)

67. Langlais, M.: Large time behavior in a nonlinear age-dependent population dynamics problem with spatial diffusion. Journal of Mathematical Biology 26(3), 319-346 (1988). DOI 10.1007/BF00277394

68. Langlais, M., Milner, F.A.: Existence and uniqueness of solutions for a diffusion model of host-parasite dynamics. Journal of Mathematical Analysis and Applications 279(2), 463 - 474 (2003). DOI 10.1016/S0022-247X(03)00020-9

69. Leksa, V., Godar, S., Cebecauer, M., et al.: The N Terminus of Mannose 6Phosphate/Insulin-like Growth Factor 2 Receptor in Regulation of Fibrinolysis and Cell Migration. Journal of Biological Chemistry 277(43), 40,575-40,582 (2002)

70. Li, Y., Cozzi, P.: Targeting uPA/uPAR in prostate cancer. Cancer Treatment Reviews 33(6), 521-527 (2007)

71. Liang, X., Yang, X., Tang, Y., et al.: RNAi-mediated downregulation of urokinase plasminogen activator receptor inhibits proliferation, adhesion, migration and invasion in oral cancer cells. Oral Oncology 44(12), 1172-1180 (2008)

72. Liu, D., Ghiso, J.A., Estrada, Y., et al.: EGFR is a transducer of the urokinase receptor initiated signal that is required for in vivo growth of a human carcinoma. Cancer Cell $\mathbf{1}(5), 445-457$ (2002)

73. Lorz, A., Lorenzi, T., Hochberg, M.E., Clairambault, J., Perthame, B.: Populational adaptive evolution, chemotherapeutic resistance and multiple anti-cancer therapies. ESAIM: Mathematical Modelling and Numerical Analysis 47, 377-399 (2013). DOI $10.1051 / \mathrm{m} 2 \mathrm{an} / 2012031$

74. MacCamy, R.: A population model with nonlinear diffusion. Journal of Differential Equations 39(1), 52 - 72 (1981). DOI 10.1016/0022-0396(81)90083-8

75. Madsen, D.H., Engelholm, L.H., Ingvarsen, S., et al.: Extracellular Collagenases and the Endocytic Receptor, Urokinase Plasminogen Activator Receptor-associated Protein/Endo180, Cooperate in Fibroblast-mediated Collagen Degradation. Journal of Biological Chemistry 282(37), 27,037-27,045 (2007)

76. Magal, P., Ruan, S. (eds.): Structured Population Models in Biology and Epidemiology. Springer-Verlag (2008)

77. Meinzer, H., Sandblad, B.: A simulation model for studies of intestine cell dynamics. Computer Methods and Programs in Biomedicine 21(2), 89-98 (1985)

78. Metz, J.A.J., Diekmann, O.: A Gentle Introduction to Structured Population Models: Three Worked Examples, Lecture Notes in Biomethamatics, vol. 68, chap. The Dynamics of Physiologically Structured Populations, pp. 3-45. Springer Berlin Heidelberg (1986)

79. Murray, J.D., Oster, G.F.: Cell traction models for generating pattern and form in morphogenesis. Journal of Mathematical Biology 19(3), 265-279 (1984)

80. Peng, L., Trucu, D., Lin, P., et al.: A multiscale mathematical model of tumour invasive growth. Bulletin of Mathematical Biology pp. 389-429 (2017). DOI $10.1007 / \mathrm{s} 11538-016-0237-2$

81. Peng, P.L., Hsieh, Y.S., Wang, C.J., et al.: Inhibitory effect of berberine on the invasion of human lung cancer cells via decreased productions of urokinase-plasminogen activator and matrix metalloproteinase-2. Toxicology and Applied Pharmacology 214(1), 8-15 (2006)

82. Persson, M., Madsen, J., Østergaard, S., et al.: 68Ga-labeling and in vivo evaluation of a uPAR binding DOTA- and NODAGA-conjugated peptide for PET imaging of invasive cancers. Nuclear Medicine and Biology 39(4), 560-569 (2012)

83. Perthame, B.: Transport Equations in Biology, chap. Population balance equations: size structure, pp. 81-110. Birkhauser Basel, Basel (2007)

84. Prigogine, I., Lefever, R.: Stability problems in cancer growth and nucleation. Comparative Biochemistry and Physiology Part B: Comparative Biochemistry 67(3), 389-393 (1980)

85. Rhandi, A.: Positivity and stability for a population equation with diffusion on $l^{1}$. Positivity 2(2), 101-113 (1998). DOI 10.1023/A:1009721915101

86. Rijken, D.C.: 2 Plasminogen activators and plasminogen activator inhibitors: biochemical aspects. Bailliere's Clinical Haematology 8(2), 291-312 (1995) 
87. de Roos, A.M.: A gentle introduction to physiologically structured population models. In: S. Tuljapurkar, H. Caswell (eds.) Structured-Population Models in Marine, Terrestrial, and Freshwater Systems, Population and Community Biology Series, vol. 18, pp. 119-204. Springer US (1997). DOI 10.1007/978-1-4615-5973-3_5

88. Sinko, J.W., Streifer, W.: A new model for age-size structure of a population. Ecology 48(6), 910-918 (1967). DOI 10.2307/1934533

89. Smith, H.W., Marshall, C.J.: Regulation of cell signalling by uPAR. Nature Reviews Molecular Cell Biology 11(1), 23-36 (2010)

90. So, J.W.H., Wu, J., Zou, X.: A reaction-diffusion model for a single species with age structure. i travelling wavefronts on unbounded domains. Proceedings of the Royal Society of London. Series A: Mathematical, Physical and Engineering Sciences 457(2012), 1841-1853 (2001). DOI 10.1098/rspa.2001.0789

91. Stillfried, G.E., Saunders, D.N., Ranson, M., et al.: Plasminogen binding and activation at the breast cancer cell surface: the integral role of urokinase activity. Breast Cancer Research 9(1), R14 (2007)

92. Sugioka, K., Kodama, A., Okada, K., et al.: TGF- $\beta 2$ promotes RPE cell invasion into a collagen gel by mediating urokinase-type plasminogen activator (uPA) expression. Experimental Eye Research 115, 13-21 (2013)

93. Trucco, E.: Mathematical models for cellular systems the von foerster equation. part i. The bulletin of mathematical biophysics 27(3), 285-304 (1965). DOI 10.1007/ BF02478406

94. Trucco, E.: Mathematical models for cellular systems. the von foerster equation. part ii. The bulletin of mathematical biophysics 27(4), 449-471 (1965). DOI 10.1007/ BF02476849

95. Trucu, D., Domschke, P., Gerisch, A., Chaplain, M.A.J.: Multiscale computational modelling and analysis of cancer invasion, Springer Lecture Notes in Mathematics, CIME Foundation Subseries, vol. 2167, pp. 275-310. Springer (2017)

96. Trucu, D., Lin, P., Chaplain, M.A.J., Wang, Y.: A multiscale moving boundary model arising in cancer invasion. Multiscale Model. Simul. 11(1), 309-335 (2013)

97. Tucker, S.L., Zimmerman, S.O.: A nonlinear model of population dynamics containing an arbitrary number of continuous structure variables. SIAM Journal on Applied Mathematics 48(3), pp. 549-591 (1988). URL http://www. jstor.org/stable/2101595

98. Waltz, D.A., Chapman, H.A.: Reversible cellular adhesion to vitronectin linked to urokinase receptor occupancy. The Journal of biological chemistry 269(20), 14,746-50 (1994)

99. Waltz, D.A., Natkin, L.R., Fujita, R.M., et al.: Plasmin and plasminogen activator inhibitor type 1 promote cellular motility by regulating the interaction between the urokinase receptor and vitronectin. The Journal of clinical investigation 100(1), 58-67 (1997)

100. Wei, Y., Waltz, D.A., Rao, N., et al.: Identification of the urokinase receptor as an adhesion receptor for vitronectin. The Journal of biological chemistry 269(51), 32,3808 (1994)

101. Yamaguchi, N., Mizutani, T., Kawabata, K., Haga, H.: Leader cells regulate collective cell migration via rac activation in the downstream signaling of integrin $\beta 1$ and pi3k. Scientific Reports 5(7656), 1-8 (2015) 